\title{
Quelques propriétés globales des espaces de Riemann
}

\section{Doctoral Thesis}

Author(s):

Preissmann, Alexandre

Publication date:

1942

Permanent link:

https://doi.org/10.3929/ethz-a-000103758

Rights / license:

In Copyright - Non-Commercial Use Permitted 


\section{Quelques propriétés globales des espaces de Riemann}

\section{T H È S E}

PRÉSENTÉE A L'ÉCOLE POLYTECHNIQUE FÉDÉRALE, ZURICH, POUR L'OBTENTION DU GRADE DE DOCTEUR ìS SCIENCES MATHÉMATIQUES PAR

\section{ALEXANDRE PREISSMAN N \\ DE LA CHAUX-DE-FONDS}

Rapporteur: M. le Prof. HOPF

Corapporteur: M. le Prof. GONSETH

1942

OR EL L F U S L I A R T G R A PHIQU E S S.A., Z U R I C H 
(Extrait des Commentarii Mathematici Helvetici) 
TABLE DES MATIERES

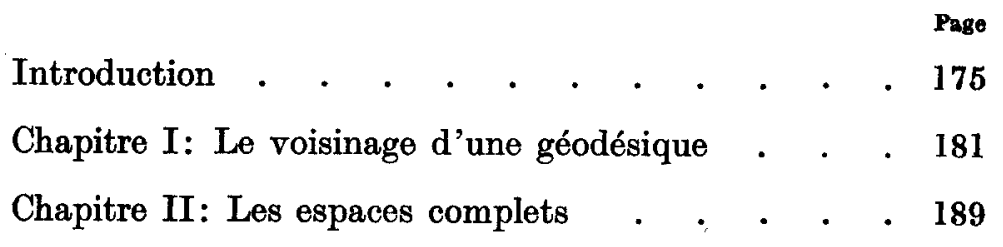

Chapitre III: Les espaces de courbure partout négative 199

Chapitre IV: Les espaces de courbure partout positive 207 


\title{
Quelques propriétés globales des espaces de Riemann
}

\author{
Par Alexandre Preissmann, Zurich
}

\section{Introduction}

\section{Le problème général}

Des recherches récentes ont eu pour but d'étendre aux variétés de Riemann des résultats connus sur les surfaces.

Comme les résultats ne sont pas encore très étendus, il va être encore possible de donner dans le présent travail une idée d'un large secteur de ces recherches ${ }^{1}$ ).

Afin de donner au problème qui sera traité ici un énoncé et une signification précis, il est nécessaire d'introduire quelques définitions.

On entend par variété topologique d̀ $n$ dimensions un espace topologique connexe tel que tout point possède un entourage homéomorphe à l'intérieur d'une sphère de l'espace euclidien à $n$ dimensions. On peut alors recouvrir une telle variété par des systèmes de coordonnées cartésiennes qui soient définis chacun pour une portion de variété et qui empiètent les uns sur les autres $x^{1}, x^{2}, \ldots, x^{n}$. Si en tout point où deux systèmes se recouvrent la transformation des coordonnées est analytique et de déterminant fonctionnel non-nul on dit que la variété est analytique.

On passe aux variétés analytiques de Riemann en donnant pour chacun des systèmes de coordonnées des fonctions analytiques $g_{i j}$ telles que la forme $g_{i j} d x^{i} d x^{j}$ soit définie positive et invariante quand on passe d'un système de coordonnées à un autre. On définit alors la longueur d'un arc de courbe $P_{1} P_{2}$ par l'expression:

$$
\int_{\dot{P}_{1}}^{P_{2}}\left(g_{i j} d x^{i} d x^{j}\right)^{\frac{1}{2}}
$$

où l'intégrale est prise sur la courbe.

On métrise les espaces de Riemann en définissant comme distance de deux points la borne inférieure de la longueur des courbes qui joignent ces deux points. Il est clair que si les deux points sont différents la distance est positive.

1) On trouve un exposé sur le problème qui est traité ici et sur les principaux résultata obtenus dans: H.Hopf: Differentialgeometrie und topologische Gestalt; Jahresbericht der D.M. V., 41. Band (1932), 209-229. 
Nous sommes maintenant en mesure de définir, comme pour les surfaces, des caractéristiques locales de la géométrie différentielles des variétés: le transport par parallélisme, la valeur de la courbure, l'équation des lignes géodésiques, etc. Ces propriétés sont étudiées en détail en particulier dans les „Leçons sur la géométrie des espaces de Riemann" de Cartan, dont nous avons gardé les notations. Toutes les propriétés locales, dont nous pourrons avoir besoin, se trouvent expliquées dans les ,Leçons" "2).

Il ne sera question ici que des propriétés internes des espaces de Riemann, c'est-à-dire des propriétés indépendantes du fait que ces espaces puissent être plongés ou non dans un espace euclidien de dimension supérieure.

Il est possible que certains des résultats énoncés puissent être obtenus sans qu'on fasse d'hypothèses sur la nature analytique des fonctions $g_{i j}$, et qu'il suffise de supposer qu'elles sont dérivables un nombre suffisant de fois, mais cette question ne nous occupera pas ici.

Le problème général est le suivant:

Quelles sont les relations qui lient les propriétés locales d'une variété d'une part et ses propriétés globales d'autre part?

Un certain nombre de théorèmes connus fournissent déjà des réponses partielles. Ils ont été démontrés par différentes méthodes. Le but de ce travail est d'abord de démontrer ces théorèmes par une méthode unique, puis d'ajouter quelques nouveaux théorèmes démontrés d'ailleurs par la même méthode.

Suivant le point de vue d'où on l'envisage le problème général présente deux aspects principaux: le problème du prolongement et le problème de la métrisation.

Le problème du prolongement se présente comme un problème analogue au problème du prolongement des fonctions analytiques: On donne une portion suffisamment petite d'une variété, la prolonger de façon à obtenir toute une variété ${ }^{1}$ ).

Le problème de la métrisation est, dans une certaine mesure, le problème inverse: On donne une variété topologique, trouver des fonctions $g_{i j}$ de façon à métriser toute cette variété.

2) E. Cartan: Legons sur la géometrie des espaces de Riemann (Paris, 1928). (Nous abrégerons ,Leçons"). Les éléments de topologie, dont nous pourrions avoir besoin, se trouvent également expliqués dans les „Leçons“. Les désignations sont les mêmes. (Nous appelons groupe fondamental, ce qui dans les "Leçons" est appelé groupe de connexion.) 
Avant d'esquisser les principaux résultats obtenus, il faut éclaircir un point essentiel: Que doit-on entendre par l'expression: toute une variété ou bien une variété complète?

On pourrait dire: une variété complète est une variété qui ne saurait être prolongée, c'est-à-dire une variété qui ne saurait être considérée comme portion d'une variété qui la contiendrait. En tout cas, les variétés prolongeables doivent être exclues. MM. Hopf et Rinow ont atteint ce but en imposant aux variétés complètes l'une ou l'autre de quatre conditions dont ils ont démontré l'équivalence ${ }^{3}$ ). Les espaces normaux de Cartan ont la même extension que les espaces complets. Les espaces complets (normaux) forment une sous-classe véritable de la classe des espaces non-prolongeables. Il y a cependant intérêt à ne considérer que les espaces complets, et c'est ce que nous ferons ici. Dans le chapitre II nous étudierons quelques propriétés des espaces complets.

\section{Le problème de la métrisation}

Nous ne considérerons que très incidemment le problème du prolongement ${ }^{1}$ ) et c'est le problème de la métrisation qui fera l'objet du travail.

Dans le problème de la métrisation, il s'agit de tirer de la connexion topologique d'un espace de Riemann des conclusions sur les possibilités de choisir les fonctions $g_{i j}$ qui déterminent la métrique. Tous les travaux qui seront discutés ici sont consacrés au problème suivant:

De la connexion topologique d'un espace déduire des conséquences au sujet du comportement de la courbure, ou, de façon plus restrictive:

Quelles sont les variétés topologiques qu'on peut métriser par une métrique dont la courbure garde un signe constant?

Ce problème est évidemment une généralisation du problème spatial de Clifford-Klein. On peut en effet considérer les formes spatiales comme des variétés qu'on peut métriser par des métriques de courbure constante. C'est ce cas particulier qui a été traité dans les premiers travaux sur le sujet de la métrisation ${ }^{1}$.

Un autre cas particulier important qui a été abondamment traité est celui des variétés riemanniennes à deux dimensions, c'est-à-dire des surfaces. Le cas des surfaces est passablement plus facile à traiter que le cas général parce qu'on peut se servir avec avantage de la formule de

s) H. Hopf und W. Rinow: Utber den Begriff der vollständigen differentialgeometrischen Fläche, Commentarii mathematici Helvetici 3 (1931), 209-225.

La généralisation, immédiate, aux espaces à plus de deux dimensions est formulóe dans: $S$. B. Myers: Riemannian manifolds in the large. Duke mathematical Journal 1 (1935), 39-49. 
Gauss-Bonnet. Il n'existe pas pour les espaces à plus de deux dimensions d'équivalent de la formule de Gauss-Bonnet, sauf dans des cas tout-à-fait spéciaux ${ }^{1}$ ). Dans ces conditions, il est compréhensible que la plupart des résultats obtenus pour les surfaces n'aient pas pu être généralisés pour les espaces à plus de deux dimensions. La méthode qui a été employée presque exclusivement consiste dans l'étude du voisinage des géodésiques et en particulier des géodésiques minimales. C'est d'ailleurs cette même méthode qu'on applique dans le cas des surfaces pour démontrer un théorème de Bonnet ${ }^{4}$ ).

\section{Le lemme de Synge}

Le théorème de Bonnet se démontre à l'aide de l'équation de Jacobi et des théorèmes de comparaison de Sturm. Dans le cas de $n$ dimensions on peut donner du théorème de Bonnet la généralisation suivante:

Un espace complet dont la courbure est partout supérieure à un nombre positif $k$ est un espace fermé de diamètre inférieur à $\frac{\pi}{\sqrt{k}}$.

Myers $^{3}$ ) et Schoenberg ${ }^{5}$ ) ont démontré ce théorème à l'aide de généralisations des théorèmes de $\left.\operatorname{Sturm}^{6}\right)$. Il est possible aussi de ramener le cas de $n$ dimensions au cas de deux dimensions à l'aide d'un lemme qui sera employé dans toutes nos démonstrations et que nous démontrerons dans le premier chapitre: le lemme de Synge ${ }^{7}$ ).

Lemme de Synge: La courbure intrinsèque d'une portion de surface $V_{2}$ qui passe par une géodésique $g$ d'un espace de Riemann $V_{n}$, ne dépend le long de $g$ que du ruban des plans tangents le long de $g$. Cette courbure est inférieure ou égale à la courbure de $V_{n}$ pour les éléments plans tangents à $V_{2}$. Il n'y a égalité que lorsque le ruban des plans tangents peut être obtenu par le déplacement parallèle d'un vecteur le long de $g$.

4) W. Blaschke: Vorlesung über Differentialgeometrie I. (Berlin, 1930), $\S 100$. Dans les démonstrations du théorème de Bonnet antérieures à celles données par $H$. Hopf et $W$. Rinow ${ }^{3}$ ), le fait que l'espace est fermé fait partie des hypothèses. On le remplace par l'hypothèse que la surface est complète.

5) J.M. Schoenberg: Some applications of the calculus of variation to Riemannian geometry. Annals of mathematics. (2), 33, 485-495. Ici aussi on suppose que l'espace est fermé.

$\left.{ }^{8}\right)$ M. Morse: A generalization of the Sturm separation and comparison theorems. Math. Annalen 103, 59-62.

7) J.L.Synge: The first and second variations of the length in Riemannian space. Proceedings of the London math. Society 25 (1926). Nous reproduisons cet article à quelques différences près dans l'introduction des coordonnées. 
Lorsque la courbure d'un espace est partout négative, l'application du lemme de Synge montre que toute surface de cet espace qui contient une famille de géodésiques est de courbure négative. Dans le cas des espaces de courbure partout positive, la dernière partie du lemme permet de construire dans le voisinage d'un arc de géodésique une portion de surface de courbure positive.

\section{Les espaces de courbure partout positive}

La généralisation du théorème de Bonnet qui a été formulé dans le paragraphe précédent peut aussi être démontrée à l'aide du lemme de Synge, c'est cette démonstration que nous reproduirons dans le chapitre IV $\left.^{8}\right)$.

Comme corollaire indiqué par Cohn-Vossen ${ }^{9}$ ) on peut déduire le théorème:

Le groupe fondamental d'une variété fermée de courbure partout positive est fini.

Mais Synge a démontré passablement plus dans un cas particulier:

Un espace fermé orientable de dimension paire et de courbure partout positive est simplement connexe ${ }^{10}$ ).

Un corollaire immédiat est le suivant:

Un espace fermé non orientable de dimension paire et de courbure partout positive possède un groupe fondamental d'ordre 2.

Nous démontrerons par la même méthode:

Un espace fermé de dimension impaire et de courbure partout positive est orientable.

On connaît fort peu de théorèmes sur les espaces ouverts de courbure partout positive. Cohn-Vossen ${ }^{11}$ ) a donné dans le cas des surfaces des propriétés globales pour les géodésiques, mais il fait un usage constant

8) J.L. Synge: On the neighborhood of a geodesic in Riemannian space. Duke mathematical Journal I (1935), 527-537.

9) S. Cohn-Vossen: Compte-rendu de Myers (3) dans: Zentralblatt für Mathematik 11 (1935), 225-226.

10) J.L. Synge: On the connectivity of spaces of positive curvature. Quarterly journal of math. (Oxford series), 7 (1936), 316-320.

11) S.Cohn-Vossen: Kürzeste Wege und Totalkrümmung auf Flächen. Compositio mathematica. 2, 69-133. S. Cohn-Vossen: Totalkrümmung und geodätischen Linien auf einfachzusammenhängenden vollständigen Flächenstücken. Recueil mathématique de Moscou, I (43), 1936, 139—163. 
de la formule de Gauss-Bonnet pour laquelle nous n'avons pas d'équivalent. Il a aussi indiqué12) pour le cas de $n$ dimensions le théorème suivant:

Un espace ouvert de courbure partout positive n'a qu'une ,extrémité"s (pour la définition de l',,extrémité" voir le chapitre II).

Un cas particulier plus facile est celui des espaces qui possèdent un pôle, c'est-à-dire un point tel que tout arc géodésique ayant ce point comme origine soit un arc géodésique minimal. Von Mangoldt ${ }^{13}$ ) a étudié les surfaces à pôles de courbure partout positive, mais en faisant un usage constant de la formule de Gauss-Bonnet. Nous retrouverons les mêmes propriétés pour les variétés en appliquant le lemme de Synge: borné.

Les pôles d'une variété de courbure partout positive forment un ensemble

Dans une variété de courbure partout positive qui possède un pôle $P$, toute géodésique possède un point à distance minimale de $P$. A partir de ce point les deux branches de la géodésique s'éloignent de $P$ de façon monotone vers l'infini.

\section{Les espaces de courbure partout négative}

Les espaces simplement connexes de courbure partout négative ont déjà été étudiés passablement en détail. Il est aisé de démontrer que:

Un espace simplement connexe de courbure partout négative est un espace à pôle et on peut choisir comme pôle un point quelconque de l'espace. On en déduit que tout espace simplement connexe de courbure partout négative est homéomorphe à l'espace euclidien ${ }^{14}$ ).

On en déduit également que la variété simplement connexe de recouvrement de tout espace de Riemann de courbure partout négative est homéomorphe à l'espace euclidien.

Pour démontrer d'autres propriétés, nous établirons le théorème suivant:

La somme des angles d'un triangle géodésique dans un espace simplement connexe de courbure partout négative est inférieure à deux droits.

12) S. Cohn-Vossen: Vollständige Riemann'sche Räume positiver Krüm. mung. C. R. Acad. des Sciences de l'U. R. S. S. 1935, III, 387-389.

13) H.v. Mangoldt: Úber diejenigen Punkte auf positiv gekrümmten Flächen, welche die Eigenschaft haben, daB die von ihnen ausgehenden geodätischen Linien nie aufhören, kürzeste Linien zu sein, Crelles Joumal
91 (1881), 23-52.

14) „Leçons", Note III. 
En considérant le groupe fondamental comme groupe d'isométrie de l'espace simplement connexe de recouvrement et en appliquant le théorème énoncé ci-dessus on trouve les théorèmes:

Si le groupe fondamental d'une variété de courbure partout négative est cyclique, cette variété possède au plus une géodésique fermée.

Toute variété de courbure partout négative possède au plus une géodésique fermée de chaque type d'homotopie.

Dans le cas particulier des espaces fermés nous sommes sûrs de l'existence d'une géodésique fermée de chaque type d'homotopie (excepté le type nul) et nous pourrons démontrer le théorème:

Tout sous-groupe abélien du groupe fondamental d'une variété fermée de courbure partout négative est un groupe cyclique.

D'autre part nous montrerons que le groupe fondamental d'une variété fermée de courbure partout négative ne saurait être un groupe cyclique.

Ces théorèmes permettent d'exclure un grand nombre de variétés topologiques fermées de la classe des variétés topologiques métrisables par une métrique de courbure partout négative; en particulier le produit topologique de deux variétés fermées ne peut pas être métrisé par une métrique de courbure partout négative.

D'autre part ces théorèmes s'appliquent aux formes spatiales hyperboliques pour lesquelles on avait démontré le théorème:

Le groupe fondamental d'une forme spatiale hyperbolique fermée ne saurait être abélien.

\section{Chapitre premier}

\section{Le voisinage d'une géodésique}

C'est la connaissance du comportement d'un espace de Riemann dans le voisinage d'une géodésique qui permettra de déduire du signe de la courbure les propriétés topologiques de ces espaces. L'étude du voisinage d'une géodésique nécessite des coordonnées appropriées.

\section{Les coordonnées normales de Riemann ${ }^{15}$ )}

Soit $A$ un point d'un espace de Riemann à $n$ dimensions $V_{n}$ et $U$ un voisinage suffisamment petit de $A$ pour qu'il n'existe dans $U$ qu'une seule géodésique joignant $A$ à un point quelc̣onque de $U$.

15) , Leçons", chapitre IX. 
Menons par $A n$ géodésiques orthogonales: $g_{1}, g_{2}, \ldots g_{n}$ que nous appellerons les axes du système de coordonnées. Pour fixer une géodésique quelconque $g$ passant par $A$ il suffit de donner les $n$ grandeurs: $\alpha^{i}$ qu'on appelle cosinus directeurs:

$$
\alpha^{i}=\cos \left(g, g_{i}\right)
$$

les quantités $\alpha^{i}$ ne sont pas indépendantes, mais liées par la relation:

$$
\left(\alpha^{1}\right)^{2}+\left(\alpha^{2}\right)^{2}+\cdots+\left(\alpha^{n}\right)^{2}=1 .
$$

Pour fixer un point quelconque $P$ de $U$ il suffit de donner les cosinus directeurs de la géodésiques $A P$ et la distance $A P=s$.

Les coordonnées normales de Riemann sont les quantités:

$$
x^{i}=s \alpha^{i} .
$$

Si l'espace considéré est l'espace euclidien, alors les coordonnées normales de Riemann sont les coordonnées cartésiennes rectangulaires.

Avec ces coordonnées les coefficients $g_{i j}$ jouissent au point $A$ de propriétés particulières, en effet au point $A$ le carré de l'élément linéaire:

devient

$$
d s^{2}=g_{i j} d x^{i} d x^{j}
$$

d'où

$$
d s^{2}=\left(d x^{1}\right)^{2}+\left(d x^{2}\right)^{2}+\cdots+\left(d x^{n}\right)^{2}
$$

$$
g_{i j}(A)=\delta_{i j} \quad \delta_{i j}=\left\{\begin{array}{l}
0 \text { si } i \neq j \\
1 \text { si } i=j
\end{array} .\right.
$$

On vérifie de même que les premières dérivées partielles des coefficients $g_{i j}$ sont nulles au point $A$ et par conséquent aussi les symboles de Christoffel $\Gamma_{i j}^{k}$ et $\Gamma_{i j k}$ :

$$
\frac{\partial g_{i j}}{\partial x^{k}}(A)=0, \quad \Gamma_{i j}^{k}(A)=0, \quad \Gamma_{i j k}(A)=0 .
$$

\section{Les coordonnées de Fermi ${ }^{16}$ )}

Il est possible d'introduire dans le voisinage d'un arc fini, simple, de géodésique des coordonnées qui jouissent des mêmes propriétés que les coordonnées de Riemann dans le voisinage d'un point.

$\left.{ }^{16}\right)$,"Leçons'", p. 262. T. Levi-Cività: Sur l'écart géodésique. Math. Annalen 97 
Soit un arc simple de géodésique $g_{0}=A B$, entouré d'un voisinage suffisamment petit pour que par chaque point de ce voisinage qui ne soit pas sur $g_{0}$, il n'existe qu'une seule géodésique ne sortant pas de $U$ et coupant $g_{0}$ à angle droit.

Au point $A$ choisissons $n-1$ directions:

$$
\lambda_{A}^{(1)}, \quad \lambda_{A}^{(2)}, \quad \lambda_{A}^{(n-1)}
$$

perpendiculaires entre elles et perpendiculaires à $g_{0}$ et déplaçons parallèlement ces directions le long de $g_{0}$, elles resteront perpendiculaires entre elles et perpendiculaires à $g_{0}$. L'ensemble des géodésiques passant par un point quelconque $P_{0}$ de $g_{0}$ et coupant $g_{0}$ à angle droit est une variété géodésique à $n-1$ dimensions $V_{n-1}\left(P_{0}\right)$ dans laquelle nous introduisons des coordonnées de Riemann en choisissant comme axes les géodésiques dans les directions:

$$
\lambda_{P_{0}}^{(1)}, \quad \lambda_{P_{0}}^{(2)}, \ldots, \lambda_{P_{0}}^{(n-1)}
$$

Soit $P$ un point de $U$ (mais pas sur $g_{0}$ ), menons la géodésique $g$ qui coupe $g_{0}$ à angle droit en $P_{0}$. P est alors dans la variété $V_{n-1}\left(P_{0}\right)$ et aura dans cet espace les coordonnées normales $x^{1}, x^{2}, \ldots, x^{n-1}$; pour fixer définitivement $P$, il faut encore donner la distance $A P_{0}=x^{n}$. Les nouvelles coordonnées : $x^{1}, x^{2}, \ldots, x^{n}$ sont appelées les coordonnées de Fermi dans le voisinage de $g_{0}$. Elles jouissent pour tout point $P_{0}$ de $g_{0}$ des propriétés suivantes:

$$
\begin{gathered}
g_{i j}\left(P_{0}\right)=\delta_{i j} \\
\left.\frac{\partial g_{i j}}{\partial x^{k}}\left(P_{0}\right)=0, \quad \Gamma_{i j}^{k}\left(P_{0}\right)=0, \quad \Gamma_{i j k}\left(P_{0}\right)=0^{17}\right) .
\end{gathered}
$$

Comme ces égalités ont lieu quel que soit $P_{0}$, c'est-à-dire quel que soit $x^{n}$, nous aurons encore:

$$
\frac{\partial}{\partial x^{n}}\left(\frac{\partial g_{i j}}{\partial x^{k}}\right)\left(P_{0}\right)=0, \frac{\partial \Gamma_{i j}^{k}}{\partial x^{n}}\left(P_{0}\right)=0, \frac{\partial \Gamma_{i j k}}{\partial x^{n}}\left(P_{0}\right)=0
$$

\section{La deuxième variation de la longueur d'une géodésique ${ }^{8}$ )}

Soit $g_{0}$ un arc de géodésique $A B$; dans le voisinage $U$ de $g_{0}$ nous introduirons un système de coordonnées de Fermi:

$$
x^{1}, x^{2}, \ldots, x^{n}
$$

17) T. Levi-Cività ${ }^{16}$ ) p. 227. 
Soit $V_{2}$ une surface analytique passant par $g_{0}$ que nous recouvrons d'un réseau de coordonnées $u, v$ qui jouisse des propriétés suivantes: $x^{i}=x^{i}(u, v)$ est l'équation de la surface.

1. $x^{i}(u, v)$ est une fonction analytique en $u$ et $v$.

2. $x^{i}(u, 0)=0$ si $i \neq n$ et $x^{n}(u, 0)=u$. C'est-à-dire que la courbe $v=0$ est la géodésique $g_{0}$ et que le paramètre $u$ sur cette courbe est l'arc $A P_{0}$.

3. $\frac{\partial}{\partial v}\left(x^{n}(u, 0)\right)=0$, c'est-à-dire que les lignes $u=u_{0}$ coupent $g_{0}$ à angle droit.

Nous appellerons ,,courbes aux variations" les courbes: $x^{i}=x^{i}\left(u, v_{0}\right)$ où $v_{0}$ a une valeur constante. Nous comparerons, entre deux valeurs de $u: u_{1}$ et $u_{2}$, la longueur d'une de ces courbes à la longueur de $g_{0}$. Cette longueur dépend du paramètre $v$ de la famille des courbes aux variations, nous l'appellerons $L(v)$.

$$
L(v)=\int_{u_{1}}^{u_{2}}\left(g_{i j} \frac{\partial x^{i}}{\partial u} \cdot \frac{\partial x^{j}}{\partial u}\right)^{\frac{1}{2}} d u .
$$

Avant de calculer $L^{\prime}(0)$ et $L^{\prime \prime}(0)$ qui sont la première et la deuxième variation de la longueur, nous introduirons quelques notations.

Soit $X$ une fonction quelconque sur $V_{2}$ : nous désignerons par le signe • la dérivée par rapport à $u$ et par le signe ' la dérivation par rapport à $v$.

En particulier:

$$
\frac{\partial X}{\partial u}=\dot{X} \quad, \quad \frac{\partial X}{\partial v}=X^{\prime} \text {. }
$$

d'où :

$$
\xi^{i}=\dot{x}^{i}, \quad \eta^{i}=x^{i \prime}
$$

$$
\xi^{i \prime}=\dot{\eta}^{i}
$$

Les coordonnées de Fermi choisies montrent que:

La quantité:

$$
\begin{array}{cl}
\xi^{i}(u, 0)=0 & \text { pour } i \neq n \quad \xi^{n}(u, 0)=1 \\
& \eta^{n}(u, 0)=0 .
\end{array}
$$

est désignée par:

$$
\left(g_{i j} \xi^{i} \xi^{j}\right)^{\frac{1}{2}}
$$

$$
F(u, v) ; \quad F(u, 0)=1
$$


Or :

$$
F^{\prime}=\frac{\left(F^{2}\right)^{\prime}}{2 F}=\frac{1}{2 F}\left\{\frac{\partial g_{i j}}{\partial x^{h}} \eta^{h} \xi^{i} \xi^{j}+2 g_{i j} \xi^{i} \xi^{j \prime}\right\}
$$

ou bien :

$$
F^{\prime}=\frac{1}{2 F}\left\{\frac{\partial g_{i j}}{\partial x^{h}} \eta^{h} \xi^{i} \xi^{j}+2 g_{i j} \xi^{i} \dot{\eta}^{j}\right\}
$$

$F^{\prime}(u, 0)$ est nul quel que soit $u$; en effet sur $g_{0}$, en vertu des coordonnées choisies :

$$
\frac{\partial g_{i j}}{\partial x^{h}}=0
$$

L'expression $g_{i j} \xi^{i} \dot{\eta}^{j}$ se réduit lorsque $v=0$ à $\dot{\eta}^{n}$, or $\eta^{n}$ est nul quel que soit $u$ lorsque $v$ est nul, par conséquent aussi $\dot{\eta}^{n}$.

$L^{\prime}(0)$ est donc aussi nulle, ce qui provient évidemment du fait que $g_{0}$ est une géodésique.

Passons à la deuxième variation:

$$
L^{\prime \prime}(v)=\int_{u_{1}}^{u_{2}} F^{\prime \prime}(u, v) d u=\int_{u_{1}}^{u_{2}} \frac{1}{2 F}\left(F^{2}\right)^{\prime \prime} d u-\int_{u_{2}}^{u_{2}} \frac{F^{\prime 2}}{F} d u .
$$

Calculons la première intégrale:

$$
\int_{u_{1}}^{u_{2}} \frac{1}{2 F}\left\{\frac{\partial^{2} g_{i j}}{\partial x^{h} \partial x^{k}} \eta^{h} \eta^{k} \xi^{i} \xi^{j}+\frac{\partial g_{i j}}{\partial x^{h}}[\cdots]+2 g_{i j}\left[\dot{\eta} \dot{\eta}^{j}+\xi^{j} \dot{\eta}^{j^{\prime}}\right]\right\} d u .
$$

Pour $v=0$, la deuxième intégrale est nulle et il reste de la première:

$$
\int_{u_{1}}^{u_{2}}\left\{\frac{1}{2} \frac{\partial^{2} g_{i j}}{\partial x^{h} \partial x^{k}} \eta^{h} \eta^{k} \xi^{i} \xi^{j}+\sum_{i=1}^{n-1}\left(\dot{\eta}^{i}\right)^{2}+\dot{\eta}^{n^{\prime}}\right\} d u .
$$

Examinons la somme:

$$
\frac{1}{2} \frac{\partial^{2} g_{i j}}{\partial x^{h} \partial x^{k}} \eta^{h} \eta^{k} \xi^{i} \xi^{j}
$$

et remarquons que:

$$
\xi^{i}(u, 0)=0, \text { si } i \neq n, \xi^{n}(u, 0)=1, \eta^{n}(u, 0)=0 .
$$

La somme devient

$$
\frac{1}{2}-\frac{\partial^{2} g_{n n}}{\partial x^{h} \cdot \partial x^{k}} \eta^{h} \eta^{k}
$$


Si nous comparons avec l'expression du tenseur de Riemann:

$$
R_{h n, k n}=\frac{1}{2}\left\{\frac{\partial^{2} g_{n n}}{\partial x^{h} \partial x^{k}}+\frac{\partial^{2} g_{h k}}{\partial x^{n} \partial x^{n}}-\frac{\partial^{2} g_{h n}}{\partial x^{k} \partial x^{h}}-\frac{\partial^{2} g_{k n}}{\partial x^{h} \partial x^{n}}\right\}
$$

où nous avons supprimé les termes où interviennent les premières dérivées des $g_{i j}$ qui sont nulles; nous voyons que les égalités (3) nous donnent:

Autrement dit:

$$
R_{k n, k n}=\frac{1}{2} \frac{\partial^{2} g_{n n}}{\partial x^{h} \partial x^{k}} .
$$

$$
-\frac{1}{2} \frac{\partial^{2} g_{i j}}{\partial x^{h} \partial x^{k}} \eta^{h} \eta^{k} \xi^{i} \xi^{j}=-R_{h i, k j} \eta^{h} \xi^{i} \eta^{k} \xi^{j}
$$

Comme les vecteurs $\xi^{i}$ et $\eta^{i}$ sont perpendiculaires, le deuxième membre de l'égalité nous donne la courbure de l'élément plan déterminé par les vecteurs $\xi^{i}$ et $\eta^{i}$ multipliée par le produit des carrés des longueurs 1 et $\eta$ des vecteurs $\xi^{i}$ et $\eta^{i}$; nous désignerons la courbure pour l'élément plan tangent à $V_{2}$ par $K$.

$L^{\prime \prime}(0)=\int_{u_{1}}^{u_{2}}\left(-K \eta^{2}+\sum_{i=1}^{n-1}\left(\dot{\eta}^{i}\right)^{2}+\dot{\eta}^{n^{\prime}}\right) d u=\int_{u_{1}}^{u_{2}}\left(\sum_{i=1}^{n-1}\left(\dot{\eta}^{i}\right)^{2}-K \eta^{2}\right) d u+\left.\eta^{n^{\prime}}\right|_{u_{1}} ^{u_{2}}$

Nous pouvons introduire le vecteur unitaire de variation $\mu^{i}$, c'est le vecteur unitaire tangent à $V_{2}$ le long de la géodésique $g_{0}$ et perpendiculaire à $g_{0}$.

$$
\begin{aligned}
\eta^{i} & =\eta \mu^{i} \quad \sum_{i=1}^{n-1}\left(\mu^{i}\right)^{2}=1 \quad \sum_{i=1}^{n-1} \mu^{i} \dot{\mu}^{i}=0 \\
\dot{\eta}^{i} & =\dot{\eta} \mu^{i}+\eta \dot{\mu}^{i} \\
\sum_{i=1}^{n-1}\left(\dot{\eta}^{i}\right)^{2} & =\dot{\eta}^{2} \cdot \sum_{i=1}^{n-1}\left(u^{i}\right)^{2}+\eta^{2} \sum_{i=1}^{n-1}\left(\dot{\mu}^{i}\right)^{2}+2 \eta \dot{\eta} \sum_{i=1}^{n-1} \mu^{i} \dot{\mu}^{i}=\dot{\eta}^{2}+\eta^{2} \sum_{i=1}^{n-1}\left(\dot{\mu}^{i}\right)^{2} .
\end{aligned}
$$

En remplaçant dans l'expression de la deuxième variation

$$
L^{\prime \prime}(0)=\int_{u_{1}}^{u_{2}}\left(\dot{\eta}^{2}+\eta^{2}\left[\sum_{i=1}^{n-1}\left(\dot{\mu}^{i}\right)-K\right]\right) d u+\left.\eta^{n^{\prime}}\right|_{u_{1}} ^{u_{2}} .
$$

Quant $\dot{\mu}^{i}$ est nul, c'est-à-dire si $\mu^{i}$ est transporté parallèlement à lui-même, alors l'expression de la deuxième variation devient:

$$
L^{\prime \prime}(0)=\int_{u_{1}}^{u_{2}}\left(\dot{\eta}^{2}-K \eta^{2}\right) d u+\left.\eta^{n^{\prime}}\right|_{u_{1}} ^{u_{2}} .
$$

18) , Leçons", p. 186. 
Que se passe-t-il si l'on considère comme courbes aux variations les courbes passant par les deux points fixes $P_{1}$ et $P_{2}$ ?

Quelle est la deuxième variation de la longueur?

$$
L^{\prime \prime}(0)=\int_{u_{1}}^{u_{2}}\left\{\dot{\eta}^{2}-\left(K-\sum_{i=1}^{n-1}\left(\dot{\mu}^{i}\right)^{2}\right) \eta^{2}\right\} d u .
$$

En effet, $\eta^{n}$ est nul pour $u_{1}$ et $u_{2}$ quel que soit $v$, donc:

$$
\eta^{n^{\prime}}\left(u_{1}, 0\right)=\eta^{n^{\prime}}\left(u_{2}, 0\right)=0
$$

Les formules (1), (2), (3) joueront un grand rôle par la suite, nous les appellerons les formules de la deuxième variation.

\section{Le lemme de Synge}

Nous pouvons introduire sur la surface $V_{2}$ donnée par les équations:

$$
x^{i}=x^{i}(u, v)
$$

dans laquelle l'élément linéaire est donné par l'élément linéaire correspondant dans $V_{n}$, un système de coordonnées de Fermi.

Nous pouvons aussi calculer la deuxième variation en nous plaçant dans le cas où les extrémités sont fixes et nous trouvons:

$$
L^{\prime \prime}(0)=\int_{u_{1}}^{u_{2}}\left(\dot{\eta}^{2}-K_{0} \eta^{2}\right) d u
$$

où $K_{0}$ désigne la courbure intrinsèque de la surface $V_{2}$.

En effet, sur une surface, tout vecteur déplacé de façon à rester perpendiculaire à une géodésique est déplacé parallélement à lui-même, par conséquent $\dot{\mu}^{i}$ est nul.

En comparant ce résultat avec celui de la formule (3), on trouve

$$
K-\sum_{i=1}^{n-1}\left(\dot{\mu}^{i}\right)^{2}=K_{0}
$$

C'est cette égalité qui exprime le lemme de Synge; on en tire:

$$
K_{\mathbf{0}} \leqslant K
$$

C'est-à-dire que la courbure gaussienne intrinsèque de la variété $V_{2}$ le long de la géodésique $g_{0}$ est inférieure ou égale à la courbure riemannienne de $V_{n}$ pour le même élément plan. 
L'égalité n'a lieu que si $\dot{\mu}^{i}$ est nul, c'est-à-dire si le plan tangent à $V_{2}$ le long de $g_{0}$ est obtenu par le déplacement parallèle d'un vecteur le long de la géodésique $g_{0}{ }^{19}$ ).

Nous voyons donc en particulier que la courbure d'une surface qui passe par une géodésique $g_{0}$ d'un espace de Riemann $V_{n}$ ne dépend le long de $g_{0}$ que du ruban des plans tangents. Il est done légitime de parler de la courbure d'un tel ruban.

Dans l'espace euclidien à trois dimensions, les remarques précédentes impliquent le théorème:

Deux surfaces qui se touchent le long d'une droite ont la même courbure le long de cette droite.

En particulier les surfaces qui, comme les surfaces développables, touchent un plan tout le long d'une droite ont aux points de contract une courbure gaussienne nulle.

La formule:

devient ici :

$$
K-\sum_{i=1}^{n-1}\left(\dot{\mu}^{i}\right)^{2}=K_{0}
$$

$$
K_{0}=-\left(\dot{\mu}^{1}\right)^{2}-\left(\mu^{2}\right)^{2} .
$$

$\mu^{i}$ désigne le vecteur unitaire tangent à la surface et perpendiculaire à la droite de contact $d$, les composantes $\mu^{1}$ et $\mu^{2}$ sont les projections du vecteur $\mu^{i}$ sur deux droites quelconques perpendiculaires à $d$ et perpendiculaires entre elles. Désignons par $\alpha$ l'angle de $\mu^{i}$ avec une direction fixe perpendiculaire à $d$, nous aurons:

$$
K_{0}=-\sin ^{2} \alpha \cdot \alpha^{2}-\cos ^{2} \alpha \cdot \dot{\alpha}^{2}=-\dot{\alpha}^{2}
$$

qui est une expression de la courbure gaussienne d'une surface passant par une droite, cette courbure est négative ou nulle.

Revenons au cas général: Dans bien des cas le lemme de Synge permet de ramener le problème du minimum de la distance dans un $V_{n}$ au problème du minimum de la distance dans une surface $V_{2}$.

Or, dans le cas des surfaces, nous connaissons les propriétés minimales des géodésiques:

L'arc de géodésique joignant deux points $A$ et $B$ tels que toute solution de l'équation de Jacobi :

$$
\ddot{y}+K_{0} y=0
$$

10) Ces propribtés pourraient aussi être tirées de théorèmes exposés dans les „Leçons“" p. 196. 
$\left(K_{0}\right.$ représente la courbure de la surface, la dérivée indiquée par le signe $\cdot$ est prise par rapport à l'arc compté sur la géodésique) ne possède qu'au plus une racine dans l'intervalle $A B$ est un arc géodésique qui réalise le minimum relatif de la distance, c'est-à-dire que cet arc est plus court que toute autre courbe joignant $A$ et $B$ et située dans un certain voisinage de la géodésique.

S'il existe une solution de l'équation de Jacobi qui possède deux racines à l'intérieur de l'intervalle $A B$, alors l'arc de géodésique $A B$ n'est certainement pas le plus court chemin entre $A$ et $B{ }^{4}$ ).

\section{Chapitre deuxième}

\section{Les espaces complets}

Quand nous cherchons à déduire des propriétés globales de toute une variété des conséquences sur le comportement de la courbure, il est clair que nous pensons à une variété non prolongeable, c'est-à-dire à une variété qui ne puisse pas être considérée comme une portion d'une variété plus grande. Toutes les variétés dont les propriétés globales interviendront ici seront des variétés non-prolongeables. Mais cette classe eșt encore trop grande pour le but que nous nous proposons.

\section{Définitions}

Les espaces qui interviendront ici sont des espaces complets au sens de MM. Hopf et Rinow que Cartan appelle des espaces normaux ${ }^{3}$ ).

Les espaces complets sont astreints à l'une quelconques des quatre conditions suivantes qui sont équivalentes entre elles:

1. Le postulat du report. On peut reporter sur chaque rayon géodésique toute longueur à partir de son origine.

2. Le postulat d'infinité. Toute ligne divergente est infiniment longue (on entend par ligne divergente l'image univoque et continue d'un rayon dans le cas où à toute suite divergente du rayon il correspond une suite de points divergente dans la variété).

Les postulats suivants se rapportent à la variété considérée comme espace métrique. On peut en effet toujours considérer un espace de Riemann comme un espace métrique en définissant la distance de deux points comme la borne inférieure de la longueur des courbes qui joignent ces deux points. 
3. Le postulat de Cauchy: Toute suite fondamentale de Cauchy converge.

4. Le postulat de compacité: Tout ensemble borné est un ensemble compact.

\section{L'espace simplement connexe de recouvrement}

Dans le cas où la variété n'est pas simplement connexe, il arrivera que nous ayons besoin de nous servir de la variété simplement connexe de recouvrement, c'est pourquoi nous rappellerons certaines propriétés de la variété simplement connexe de recouvrement. A chaque point de la variété simplement connexe de recouvrement $\bar{V}_{n}$ correspond un seul point de $V_{n}$, mais à chaque point de $V_{n}$ correspondent en général plusieurs points de $\bar{V}_{n}$. A une courbe fermée de $V_{n}$ du type d'homotopie $a$ et passant par le point $x$ correspondent des arcs de courbe joignant en particulier deux points correspondant à $x: \bar{x}^{\prime}$ et $\bar{x}^{\prime \prime}$. Si l'on choisit $\bar{x}^{\prime}$ fixe, alors $\bar{x}^{\prime \prime}$ ne dépendra pas de la forme particulière de la courbe fermée de type $a$ dans $V_{n}$. Ainsi la classe d'homotopie $a$ fait correspondre à un point $\bar{x}^{\prime}$ de l'espace simplement connexe de recouvrement un point $\bar{x}^{\prime \prime}$ qui est l'image du même point de $V_{n}$. Comme $\bar{x}^{\prime \prime}$ ne dépend que de $a$ et de $\bar{x}^{\prime}$, nous écrirons: $\bar{x}^{\prime \prime}=a\left(\bar{x}^{\prime}\right)$.

A chaque élément du groupe fondamental correspond donc une transformation de l'espace simplement connexe de recouvrement sur luimême. Cette transformation est une transformation sans points doubles et les points qui se correspondent dans cette transformation sont des images du même point de $V_{n}$. Si nous douons la variété simplement connexe de recouvrement dans l'entourage quelconque d'un point de la même métrique que celle de l'entourage correspondant de $V_{n}$, nous avons métrisé $\bar{V}_{n}$, et il s'ensuit que la transformation qui correspond à l'élément $a$ est une isométrie. Nous appellerons également $a$ l'isométrie qui correspond à l'élément $a$ du groupe d'homotopie.

Encore une remarque: Considérons dans $V_{n}$ une ligne fermée de type $a$ indéfiniment parcourue dans les deux sens et passant par un point $x$, il lui correspondra dans $\bar{V}_{n}$ en particulier une ligne passant par un point $\bar{x}$ et aussi par les points $a^{k}(\bar{x})$, la ligne est fermée si l'élément $a$ est d'ordre fini dans le groupe d'homotopie, elle est ouverte (et divergente) si $a$ est d'ordre infini.

Si $V_{n}$ est un espace normal, il satisfait au postulat 3 (par exemple); et il est aisé de voir qu'alors $\bar{V}_{n}$ satisfait aussi au postulat 3 . Donc:

La variété simplement connexe de recouvrement d'un espace complet est aussi un espace complet. 


\section{Un theorème fondamental}

MM. Hopf et Rinow ${ }^{3}$ ) ont démontré pour les espaces de Riemann un théorème qui n'est pas vrai pour tous les espaces non prolongeables et qui sera très souvent appliqué dans la suite:

Théorème fondamental des variétés complètes: On peut joindre deux points quelconques d'une variété complète par un arc géodésique minimal

\section{Une application aux espaces non simplement connexes}

Nous allons démontrer le théorème suivant que nous appellerons le lemme 1 .

Lemme 1: A chaque élément $a(\neq e)$ du groupe fondamental d'un espace fermé non simplement connexe correspond une ligne géodésique fermée du type $a$, qui réalise le minimum de la longueur des courbes fermées du type $a$.

Démonstration: Soit $\bar{V}_{n}$ l'espace simplement connexe de recouvrement de $V_{n}$ et soit $V_{n}^{\prime}$ un domaine fondamental de $V_{n}$ dans $\bar{V}_{n}$. Tout élément $a(\neq e)$ fait correspondre à tout point $\bar{x}^{\prime}$ de $V_{n}^{\prime}$ un point $\bar{x}^{\prime \prime}=a\left(\bar{x}^{\prime}\right)$. Toute ligne joignant $\bar{x}^{\prime}$ à $\bar{x}^{\prime \prime}$ correspond dans l'espace $V_{n} \grave{a}$ une courbe de type $a$ et passant par $x$ et réciproquement. Or dans $\bar{V}_{n}$ la distance $l\left(\bar{x}^{\prime}, \bar{x}^{\prime \prime}\right)$ est une fonction continue des variables $\bar{x}^{\prime}$ et $\bar{x}^{\prime \prime}$, comme $\bar{x}^{\prime \prime}$ est une fonction continue de $\bar{x}^{\prime}, l\left(\bar{x}^{\prime}, \bar{x}^{\prime \prime}\right)$ sera aussi une fonction continue de $\bar{x}^{\prime}$. Comme $V_{n}^{\prime}$ est un domaine compact, la fonction $l\left(\bar{x}^{\prime}, \bar{x}^{\prime \prime}\right)$ atteint son minimum au moins pour un point $\bar{\xi}$. Construisons un arc géodésique $(\bar{\xi}, a(\bar{\xi}))$ qui réalise la distance $l(\bar{\xi}, a(\bar{\xi}))$. (Cet arc minimum existe en vertu du théorème fondamental sur les espaces complets.) Dans $V_{n}$ cet arc correspond à un arc géodésique fermé qui fait éventuellement un angle en $\xi$. Nous allons montrer que cette éventualité ne se présente pas, c'est-à-dire que nous avons construit une géodésique fermée.

Supposons en effet que l'arc géodésique considéré ait un angle en $\xi$, nous pouvons choisir sur les deux côtés de l'angle deux points $x_{1}$ et $x_{2}$ suffisamment rapprochés de $\xi$ pour que le petit triangle $x_{1} \xi x_{2}$ soit homotope 0 . Dans ces conditions le cycle géodésique $x_{1} M x_{2} x_{1}$ est du même type $a$ que l'arc géodésique $\xi x_{1} M x_{2} \xi$. Or l'inégalité du triangle montre que le second cycle est plus long que le premier; l'arc géodésique $\xi M \xi$ ne représenterait pas alors le minimum de la longueur des courbes de son type.

Il existe donc pour chaque type $a$ une courbe de longueur minimale qui est une géodésique fermée. 


\section{Les rayons géodésiques minimaux et les extrémités des espaces ouverts}

Nous appellerons rayon géodésique minimal un rayon géodésique qui représente la plus courte distance entre son origine et chacun de ses points. Contrairement à ce qui se passe pour les espaces fermés, $R_{i n o w}{ }^{20}$ ) et Myers ${ }^{3}$ ) ont montré que dans un espace ouvert, il existe par chaque point au moins un rayon géodésique minimal. Nous allons donner une esquisse de cette démonstration.

Soit $A$ un point quelconque de la variété ouverte $V_{n}$ et

$$
P_{1}, P_{2}, \ldots, P_{m}, \ldots
$$

une suite divergente de points; nous construisons les géodésiques:

$$
g_{1}=A P_{1}, g_{2}=A P_{2}, \ldots, g_{m}=A P_{m}, \ldots
$$

qui donnent les plus courtes distances entre leurs extrémités.

L'ensemble des directions des géodésiques $g_{1}, g_{2}, \ldots, g_{m}$ en $A$ possède au moins une direction d'accumulation, il est facile de voir que la géodésique ayant cette direction au point $A$ est une géodésique minimale. Nous avons ainsi construit un rayon géodésique minimal par le point quelconque $A$. Il est possible qu'il n'en existe qu'un. On voit facilement que l'ensemble des directions des rayons géodésiques minimaux par un point est un ensemble fermé.

Une question en rapport avec celle des rayons géodésiques minimaux est celle des extrémités des espaces ouverts ${ }^{21}$ ).

Explication: Nous dirons qu'une suite de points:

$$
P_{1}, P_{2}, \ldots, P_{m}, \ldots
$$

diverge vers une extrémité, si, quelles que soient les suites partielles:

$$
\begin{aligned}
& Q_{1}, Q_{2}, \ldots, Q_{m}, \ldots \\
& S_{1}, S_{2}, \ldots, S_{m}, \ldots
\end{aligned}
$$

extraites de la suite (1) et quel que soit le domaine compact $D$, il est possible de trouver des courbes joignant:

$$
Q_{1} \text { à } S_{1}, Q_{2} \text { à } S_{2}, \ldots, Q_{m} \text { à } S_{m}, \ldots
$$

20) W. Rinow: UUber Zusammenhänge der Differentialgeometrie im GroBen und im Kleinen. Mathematische Zeitschrift 35 (1932). Rinow a démontré le théorème pour les surfaces, Myers l'a généralisé pour les variétés.

21) H. Freudenthal: Über die Enden topologischer Räume und Gruppen. Math. Zeitschrift 33 (1931), 692-713. 
qui n'aient, à un nombre fini d'exceptions près, aucun point commun avec le domaine $D$.

Dans tout espace riemannien ouvert il existe une suite de points qui diverge vers une extrémité. L'exemple est fourni par, une suite divergente de points sur un rayon géodésique minimal $g$ d'origine $A$. Soient en effet deux suites partielles de cette suite:

nous joignons:

$$
\begin{aligned}
& Q_{1}, Q_{2}, \ldots, Q_{m}, \ldots \\
& S_{1}, S_{2}, \ldots, S_{m}, \ldots
\end{aligned}
$$

$$
Q_{1} \grave{\mathrm{a}} S_{1}, Q_{2} \text { à } S_{2}, \ldots, Q_{m} \text { à } S_{m}, \ldots
$$

par les arcs de géodésiques minimaux qui sont des arcs de $g$. A cause de la propriété minimale de $g$, les points de l'arc $Q_{n} S_{m}$ sont à une distance de $A$ comprise entre les distances $A$ et $A S_{m}$ qui toutes deux tendent vers l'infini avec $m$. A partir d'un certain rang ces arcs n'ont aucun point commun avec un domaine compact donné quelconque. Dans ces conditions nous dirons que le rayon géodésique $g$ diverge vers une extrémité.

Explication: Nous dirons que deux suites de points:

$$
\begin{gathered}
P_{1}, P_{2}, \ldots, P_{m}, \ldots \\
Q_{1}, Q_{2}, \ldots, Q_{m}, \ldots
\end{gathered}
$$

divergent vers la même extrémité, si la suite:

$$
P_{1}, Q_{1}, P_{2}, Q_{2}, \ldots, P_{m}, Q_{m}, \ldots
$$

diverge vers une extrémité.

Si les deux suites:

$$
\begin{gathered}
P_{1}, P_{2}, \ldots, P_{m}, \ldots \\
Q_{1}, Q_{2}, \ldots, Q_{m}, \ldots
\end{gathered}
$$

divergent l'une et l'autre vers une extrémité, mais ne divergent pas vers la même extrémité (nous dirons alors que les deux suites divergent vers des extrémités différentes), alors il existe un domaine compact $D$ tel que parmi les arcs quelconques joignant:

$$
P_{1} \text { à } Q_{1}, P_{2} \text { à } Q_{2}, \ldots, P_{m} \text { à } Q_{m}, \ldots
$$

il en existe une infinité qui aient des points communs avec le domaine $D$. 
On montre que dans ces conditions il existe un domaine $D$ tel que, à un nombre fini d'exceptions près, tous les chemins joignant

$$
P_{1} \text { à } Q_{1}, P_{2} \text { à } Q_{2}, \ldots, P_{m} \text { à } Q_{m}, \ldots
$$

ont un point commun avec le domaine $D$.

Un espace n'a qu'une extrémité si toute suite divergente diverge vers une extrémité.

Exemple: l'espace euclidien.

Un espace a deux extrémités s'il existe deux suites $P_{i}$ et $Q_{i}$ qui divergent vers deux extrémités différentes et si toute suite divergeant vers une extrémité diverge, soit vers l'extrémité de la suite $P_{i}$, soit vers l'extrémité de la suite $Q_{i}$.

Exemple: un cylindre circulaire.

On définit de même les espaces à $3,4, \ldots, n$ extrémités. Il peut exister des espaces à une infinité d'extrémités.

On démontre qu'il existe au moins un rayon géodésique minimal issu d'un point quelconque $A$ qui tend vers une extrémité choisie à l'avance. On le construit en choisissant une suite divergeant vers l'extrémité donnée:

$$
P_{1}, P_{2}, \ldots, P_{m}, \ldots
$$

et en menant les ares géodésiques minimaux:

$$
A P_{1}, A P_{2}, \ldots, A P_{m}, \ldots \text {. }
$$

Les directions de ces arcs géodésiques ont en $A$ au moins une direction d'accumulation. Le rayon géodésique $g$ ayant une direction d'accumulation comme tangente en $A$ est un rayon géodésique minimal qui tend vers la même extrémité que la suite $P_{i}$.

En effet, nous savons que ce rayon est un rayon géodésique minimal. Soit $D$ un domaine compact quelconque, nous pouvons construire une Sphère $S$ qui contienne $D$.

Pour $n$ suffisamment grand les rayons géodésiques $A P_{n}$ coupent $S$ en des points $Q_{n}$ dont le point d'intersection $Q$ de $g$ avec $S$ est un point d'accumulation. (Les points $Q_{n}$ dépendent de façon continue de la direction des géodésiques $A Q_{n}$ en $A$.) Si $Q_{n}$ est suffisamment près de $Q$ on peut joindre ces deux points par une courbe sur la frontière de la sphère $S$.

Soit $P_{i_{1}}, \ldots, P_{j_{n}}, \ldots$ une suite partielle de la suite $P_{i}$ telle que les $Q_{j_{i}}$ correspondants tendent vers $Q$. A partir d'un certain $j_{n}$ on peut 
joindre $P_{j_{n}}$ à un point quelconque $P$ de $g$ en dehors de $S$ de la façon suivante: de $P_{j_{n}}$ à $Q_{j_{n}}$ sur l'arc géodésique minimal $A P_{j_{n}}$, de $Q_{j_{n}}$ à $Q$ sur la frontière de $S$, de $Q$ à $P$ sur l'arc $g$. Nous avons ainsi évité le domaine $D$. Une suite divergente sur $g$ tend donc vers la même extrémité que la suite $P_{j_{n}}$, donc que la suite $P_{i}$.

\section{Les points conjugués sur une géodésique et les espaces à pôles}

La méthode que nous employerons habituellement pour répondre à la question:

„L'arc géodésique $z=A B$ représente-t-il le plus court chemin entre $A$ et $B$ pour des variations suffisamment petites de l'arc géodésique? (minimum relatif de la distance)."

consiste à ramener ce problème, grâce au lemme de Synge, au problème équivalent pour les surfaces.

Il est possible cependant de traiter ce problème directement selon une méthode que nous allons esquisser et que Myers ${ }^{3}$ ) a spécialement employée ${ }^{22}$ ).

Le but des considérations qui vont suivre est de montrer qu'on peut définir les points conjugués sur une géodésique de la même façon que pour les surfaces et qu'il est possible de démontrer le théorème suivant:

Si $B$ n'est pas un point conjugué de $A$ sur une géodésique $g$, il est possible d'introduire dans le voisinage de $B$ des coordonnées normales de Riemann de centre $A$.

La deuxième variation de la longueur d'une géodésique est:

$$
L^{\prime \prime}(0)=\int_{u(A)}^{u(B)}\left(R_{i n, j n} \eta^{i} \eta^{j}+\Sigma\left(\dot{\eta}^{i}\right)^{2}\right) d u+\left.\eta^{n^{\prime}}\right|_{u(A)} ^{u(B)}
$$

où les différentes lettres ont la même signification que dans le chapitre I et où en particulier les coordonnées choisies sont des coordonnées de Fermi. Les équations différentielles qui correspondent à l'équation de Jacobi dans le cas des surfaces sont alors:

$$
\ddot{\eta}^{i}-R_{i n, j n} \eta^{j}=0 \text {. }
$$

On dit que $A$ et $A^{\prime}$ sont des points conjugués si une solution des équations (2) s'annulle pour $A$ et $A^{\prime}$.

On montre que les équations (2) jouissent de propriétés analogues à celles des équations de Jacobi, c'est-à-dire:

22) Voir, à part Myers $^{3}$ ), Levi-Cività ${ }^{16}$ ) où l'on trouve des compléments sur le sujet traité ici. 
S'il n'existe pas sur l'arc $A B$ de point conjugué de $A$, alors l'arc $A B$ est un minimum relatif de la distance entre $A$ et $B$; s'il existe sur l'arc $A B$ un point $A^{\prime}$ conjugué de $A$, alors l'arc $A B$ ne représente pas un minimum relatif de la distance entre $A$ et $B$.

Les équations (2) ont encore une autre signification. Considérons une famille de géodésiques de paramètre $\alpha$ :

$$
\begin{aligned}
x^{i} & =x^{i}(u, \alpha) \quad 1 \leqq i \leqq n-1 \\
x^{n} & =u
\end{aligned}
$$

dans le voisinage de $g=A B\left\{u(A)=0, u(B)=u_{0}\right\}$ telle que $x^{i}(u, 0)=0$ $i \neq n$, c'est-à-dire que la géodésique de la famille qui correspond à la valeur $\alpha=0$ soit $g$. On montre que les dérivées $x_{\alpha}^{i}$ satisfont aux équations différentielles linéaires (2) quelle que soit la famille choisie. On appelle les équations (2) aussi les équations différentielles des géodésiques voisines de $g$.

Quelle est dans cette nouvelle interprétation la signification des points conjugués?

Considérons encore l'arc géodésique $g$ et les géodésiques voisines de $g$ passant par $A$. Nous pouvons déterminer ces géodésiques par les cosinus angulaires au point $A$, c'est-à-dire par les valeurs des dérivées $\dot{x}^{i}(0)\{i \neq n\}$.

Les valeurs $x^{i}\left(u_{0}\right)$ sont des fonctions des valeurs $x^{i}(0)$, nous avons les relations:

$$
\dot{x}^{i}\left(u_{0}\right)=f^{i}\left(\dot{x}^{n}(0)\right) \quad i=1, \ldots, n-1, k=1, \ldots, n-1 .
$$

Considérons d'autre part les solutions des équations différentielles (2) déterminées par les conditions initiales:

$$
\eta^{i}(0)=0 \quad i=1, \ldots, n-1 .
$$

Nous avons une relation linéaire entre les valeurs $\eta^{i}\left(u_{0}\right)$ et $\dot{\eta}^{i}(0)$

$$
\eta^{i}\left(u_{0}\right)=\sum_{k=1}^{n-1} c_{i k} \dot{\eta}^{k}(0) \quad i=1,2, \ldots, n-1 .
$$

On montre que le déterminant fonctionnel des équations (3) est égal au déterminant fonctionnel des équations (4) pour $x^{i}=0$. Or le déterminant fonctionnel des équations (4) s'annulle précisément dans le cas où les équations linéaires (4) sont dégénérées, mais cela signifie précisément que $B$ est un point conjugué de $A$ et réciproquement.

Si $B$ n'est pas conjugué de $A$, il existe une relation biunivoque entre les valeurs $x^{i}\left(u_{0}\right)$ et les valeurs $\dot{x}^{i}(0)$ lorsque ces dernières valeurs sont suffisamment petites. 
Sur la variété géodésique formée par toutes les géodésiques normales à $g$ en $B$, il existe donc pour chaque point $P$ dans un certain voisinage de $B$ une et une seule ligne géodésique issue de $A$ et passant par $P$, et l'on peut étendre cette correspondance à tout un voisinage de $B$. Autrement dit on peut introduire dans le voisinage de $B$ des coordonnées de Riemann de centre A .

Un cas particulier important. Nous examinerons un cas qui va se présenter assez souvent, c'est celui où il existe un point $P$ qui jouisse de la propriété suivante:

Le point $P$ ne possède de point conjugué sur aucune géodésique, autrement dit, les rayons géodésiques issus de $P$ représentent tous un minimum relatif.

En ajoutant l'hypothèse que l'espace est simplement connexe, Cartan et Myers ont démontré qu'on peut étendre la correspondance biunivoque entre les coordonnées deFermi et les coordonnées de Riemann de centre $P$ à tout l'espace. Cela signifie que les coordonnées de Riemann de centre $P$ conviennent à tout l'espace.

Cela signifie en particulier qu'un tel espace est homéomorphe à l'espace euclidien. En effet, il suffit pour avoir une représentation topologique de l'espace $V_{n}$ sur l'espace euclidien $R_{n}$ de faire correspondre au point de $V_{n}$ dont les coordonnées de Riemann sont $x^{1}, x^{2}, \ldots x^{n}$ le point de $R_{n}$ dont les coordonnées cartésiennes sont $x^{1}, x^{2}, \ldots x^{n}$.

Une conséquence des résultats précédents est celle-ci:

Si un espace $V_{n}$ contient un point $P$ sans points conjugués, alors l'espace simplement connexe de recouvrement est homéomorphe au $R_{n}$.

Nous appellerons espace à pôle un espace qui possède un point $P$ (le pôle) tel que tous les rayons géodésiques issus de $P$ soient des rayons géodésiques minimaux. Un tel point ne possède de point conjugué sur aucune géodésique. Il est d'autre part simplement connexe. (Si un espace n'est pas simplement connexe, il existe pour tout point $Q$ un arc géodésique fermé faisant éventuellement un angle en $Q$.) Un tel espace est done homéomorphe au $R_{n}$. Nous allons souvent faire usage des dernières propriétés des espaces $\grave{a}$ pôles ${ }^{23}$ ).

7. On peut déduire d'un théorème général de $S m i t h{ }^{24}$ ) le théorème particulier:

${ }^{23}$ ) Ces résultats sur les espaces à pôles ont été exposés par Rinow ${ }^{21}$ ) et Myers ${ }^{3}$ ).

24) $P$. A. Smith: A theorem of fixed points for periodic transformations. Annals of Math. II. s. 35 (1934), 572-578. S. Eilenberg: On a theorem of P. A. Smith concerning fixed points for periodic transformations. Duke math. journal. 6 (1940). 
Toute transformation topologique de l'espace euclidien $R_{n}$ sur luimême qui est périodique possède un point fixe.

Comme le groupe fondamental d'un espace $V_{n}$ peut toujours être considéré comme un groupe d'isométrie sans points doubles de l'espace simplement connexe de recouvrement nous pouvons déduire, en nous servant des résultats du dernier paragraphe:

$S i$ un espace $V_{n}$ possède un point $P$ sans points conjugués, alors tout élément $(\neq e)$ du groupe fondamental est d'ordre infini.

\section{Les lignes géodésiques dans les espaces à pôles.}

Nous pouvons considérer un espace à pôle comme un espace euclidien doué d'une certaine métrique, et nous pouvons choisir la représentation de telle façon que les droites (au sens euclidien) issues du point $P$ (le pôle) correspondent à des géodésiques.

Dans cette représentation une ligne géodésique $g$ est, ou bien une droite passant par $P$, ou bien une courbe qui n'est tangente à aucune droite passant par $P$ (les géodésiques sont les solutions d'équations différentielles du deuxième degré). Par conséquent si l'on se borne à la deuxième possibilité, le cône de sommet $P$ et de directrice $g$ est un cône régulier (au sens euclidien) $H$.

La métrique de l'espace $V_{n}$ induit une certaine métrique sur le cône $H$; dans cette métrique, les droites passant par $P$ restent évidemment des géodésiques. Nous pouvons développer ce cône sur le plan euclidien, il est possible que le plan euclidien soit recouvert plusieurs fois ou qu'il ne soit pas recouvert tout entier. Nous appellerons $D$ la portion de surface de Riemann ainsi obtenue. Nous pouvons introduire sur $D$ un système de coordonnées polaires (au sens euclidien) de centre $P$, ce qui revient sur $H$ à un système de coordonnées polaires géodésiques.

L'élément d'arc est alors donné par la formule ${ }^{25}$ ):

$$
d s^{2}=d r^{2}+G^{2}(r, \varphi) d \varphi^{2} .
$$

La fonction $G(r, p)$ dépend de la courbure intrinsèque de $H$ de la façon suivante: Soit $K_{0}$ la courbure de $H$ le long de la géodésique $\varphi=\varphi_{0}$, $G(r, \varphi)$ satisfait à l'équation de Jacobi:

$$
G_{r r}\left(r, \varphi_{0}\right)+K_{0} G\left(r, \varphi_{0}\right)=0
$$

avec les conditions initiales: $G\left(0, \varphi_{0}\right)=0 \quad G_{r}\left(0, \varphi_{0}\right)=1$.

25) Blaschke: Differentialgeometrie I, $\$ 57$ (Berlin, 1930), nous appelons $G^{2} \mathrm{ce}$ que Blaschke désigne par $G$. 
Une géodésique $g$ de l'espace $V_{n}$ correspond naturellement aussi à une géodésique de $H$ et son équation différentielle est:

$$
\frac{d}{d \varphi}\left(\frac{d r}{d s}\right)-G_{r}\left(\frac{G d \varphi}{d s}\right)=0
$$

(Equation d'Euler-Lagrange pour le minimum de $s$ ).

Nous introduisons l'angle $\psi(0 \leqslant \psi \leqslant \pi)$ que fait la tangente à la géodésique $g$ dans le sens des $s$ croissants avec la géodésique $\varphi=\varphi_{0}$ dans le sens des $r$ croissants. On voit que:

$$
\begin{aligned}
& \sin \psi=\frac{G d \varphi}{d s} \\
& \cos \psi=\frac{d r}{d s} .
\end{aligned}
$$

Si nous introduisons cette notation, l'équation différentielle de la géodésique devient:

$$
\frac{d \psi}{d \varphi}=-G_{r}
$$

Ces résultats sont généraux, nous n'avons pas fait intervenir la courbure de l'espace $V_{n}$. Ils ne donnent d'ailleurs que l'indication du comportement de la géodésique $g$ sur le développement du cône géodésique, mais pas dans l'espace $V_{n}$.

\section{Chapitre troisième}

\section{Les espaces de courbure partout négative}

1. Le théorème fondamental sur les espaces simplement connexes de courbure partout négative ${ }^{14}$ )

Dans le cas où la courbure est partout négative, les équations (3) du chapitre I, 3 montrent que la deuxième variation de la longueur d'une géodésique est toujours positive, ce qui signifie que tout arc géodésique réalise un minimum relatif de la longueur. Choisissons un point quelconque $P$, toutes les géodésiques issues de $P$ sont des rayons géodésiques qui ne contiennent pas de points conjugués de $P$; si, en plus, l'espace est simplement connexe, les résultats de 6 , II montrent que le point $P$ est un pôle et que l'espace est homéomorphe à l'espace euclidien. 
Théorème 1: Un espace simplement connexe de courbure partout négative est homéomorphe à l'espace euclidien. ${ }^{14}$ )

Une conséquence immédiate, c'est que la variété simplement connexe de recouvrement de tout espace de courbure partout négative est un espace homéomorphe à l'espace euclidien.

Exemple: Il est impossible de recouvrir d'une métrique de courbure partout négative une variété topologique qui serait le produit topologique d'une sphère et d'un cercle. En effet, la variété simplement connexe de recouvrement est le produit topologique d'une sphère et d'une droite qui n'est pas homéomorphe à l'espace euclidien. (Elle est homéomorphe à l'espace euclidien dont on a enlevé un point.)

Une conséquence du fait qu'on peut considérer tout espace simplement connexe de courbure partout négative comme un espace à pôle, dont le pôle peut être choisi arbitrairement, c'est que:

deux points quelconques ne peuvent être joints que par une seule ligne géodésique;

en particulier il n'existe pas de géodésique fermée.

\section{L'allure des lignes géodésiques des espaces simplement connexes de courbure partout négative ${ }^{14}$ )}

Nous allons appliquer la méthode exposée dans le paragraphe 8, II à l'étude de l'allure des géodésiques dans le cas où la courbure est partout négative.

Soit $P$ un point quelconque de la variété que nous considérerons comme pôle et $g$ une géodésique quelconque ne passant pas par $P$. Nous construisons à nouveau le cône géodésique de sommet $P$ et de directrice $g$ sur le développement duquel nous introduisons un système de coordonnées polaires géodésiques. L'élément d'arc s'exprime alors par la formule:

$$
d s^{2}=d r^{2}+G^{2}(r, \varphi) d \varphi^{2}
$$

et l'équation de la géodésique $g$ sur le développement du cône géodésique est:

$$
\frac{d \psi}{d \varphi}=-G_{r}
$$

où $\psi$ représente l'angle de la géodésique $g$ (dans le sens des $s$ croissants) avec la ligne $\varphi=\varphi_{0}$ (dans le sens des $r$ croissants). Nous allons démontrer successivement les trois lemmes suivants:

Lemme 1: Dans un espace de courbure partout négative, la quantité $G_{r}$ est partout positive quelle que soit la géodésique $g$ choisie. 
Lemme 2: Si la quantité $G_{r}$ est partout positive et qu'il existe un point $M$ sur $g$ tel que $\psi=\frac{\pi}{2}$ alors les deux branches de la géodésique à partir de $M$ s'éloignent de façon monotone vers l'infini.

Lemme 3: Si la quantité $G_{r}$ est positive partout, alors il existe un point $M \operatorname{sur} g$ tel que $\psi(M)=\frac{\pi}{2}$.

Démonstration du lemme 1: Le cône géodésique que nous avons construit contient une infinité de géodésiques de $V_{n}$. Il en passe une par chaque point. D'après le lemme de Synge, la courbure intrinsèque du cône en un de ses points est inférieure ou égale à la courbure de l'espace pour le même élément plan. Par conséquent la courbure intrinsèque du cône est négative en tous ses points. Or nous savons que $G(r, \varphi)$ est la solution de l'équation de l'équation de Jacobi:

$$
G_{r r}\left(r, \varphi_{0}\right)+K_{0} G\left(r, \varphi_{0}\right)=0
$$

qui satisfait aux conditions initiales: $G\left(0, \varphi_{0}\right)=0, G_{r}\left(0, \varphi_{0}\right)=1$.

L'équation de Jacobi montre immédiatement que si $K_{0}<0, G_{r}$ croît avec $r$ pour $r>0$. Donc $G_{r}>1>0$.

Démonstration du lemme 2: L'équation:

$$
\frac{d \psi}{d \varphi}=-G_{r}
$$

appliquée au point $M$ montre immédiatement que pour un point $R$ tel que $\varphi(R)>\varphi(M)$, nous aurons $\psi(R)<\psi(M)=\frac{\pi}{2}$.

Je prétends que lorsque $s$ tend vers l'infini par valeurs positives $r$ tend aussi vers l'infini. En effet, pour $\varphi>\varphi(R)$ l'équation (1) montre que $\psi<\psi(R)$. Par conséquent:

$$
\begin{gathered}
\frac{d r}{d s}=\cos \psi>\cos \psi(R) \\
r-r(R)>\cos \psi(R)\{s-s(R)\} .
\end{gathered}
$$

Et l'on voit que $r$ tend vers l'infini avec $s$. On démontre de même que $r$ tend vers l'infini quand $s$ tend vers l'infini par valeurs négatives.

Démonstration du lemme 3: Nous allons faire la démonstration par l'absurde: Choisissons un point quelconque $Q$ sur $g$. Nous pouvons sup- 
poser que $\psi(Q)>\frac{\pi}{2}$ (sinon nous ferions croître $s$ dans le sens contraire). Supposons que lorsque $s$ augmente, $\psi$ ne devienne jamais égal à $\frac{\pi}{2}$, autrement dit, reste toujours supérieur à $\frac{\pi}{2}$. Dans ces conditions $r$ diminue constamment, en effet:

$$
\frac{d r}{d s}=\cos \psi<0
$$

tout le rayon issu de $Q$ serait donc compris dans la sphère $r \leqslant r(Q)$, il faudrait alors supposer que la géodésique $g$ fît une infinité de tours autour de $P$ dans le développement du cône géodésique (en effet, la relation:

$$
d \varphi=\frac{\sin \psi \cdot d s}{G}>\frac{\sin \psi(Q) d s}{G}
$$

et le fait que $G$ possède dans la sphère $r \leqslant r(Q)$ un certain maximum montrent que $\varphi$ croît indéfiniment quand $s$ croît indéfiniment). Comme d'autre part $G_{r}$ a dans la sphère compacte $r \leqslant r(Q)$, un certain minimum positif $\alpha$ on a:

$$
\begin{gathered}
\frac{d \psi}{d \varphi}=-G_{r}<-\alpha \\
\psi-\psi(Q)<-\alpha\{\varphi-\varphi(Q)\} .
\end{gathered}
$$

Comme on peut choisir $\varphi$ aussi grand qu'on veut, cela signifie que $\psi$ atteindra une fois la valeur $\frac{\pi}{2}$. Nous sommes arrivés à une contradiction. Le lemme 3 est donc démontré.

Les lemmes 2 et 3 ont été démontrés sans qu'on tienne compte du fait que la courbure était partout négative. Nous pourrons par conséquent les appliquer dans le chapitre suivant.

Dans le cas où la courbure est partout négative, nous avons démontré plus que le lemme 1 , nous avons démontré $G_{r}>1$.

Et alors:

$$
\frac{d \psi}{d \varphi}=-G_{r}<-1
$$

Supposons $\varphi=0$ pour le point $M$ à distance minimale de $P$. Nous voyons:

$$
|\varphi|<\left|\psi-\frac{\pi}{2}\right|
$$


Comme $\psi$ varie entre $\pi$ et $0, \varphi$ varie entre $\varphi_{0}$ et $\varphi_{1}\left(-\frac{\pi}{2}<\varphi_{0}<0\right.$, $\left.0<\varphi_{1}<\frac{\pi}{2}\right)$.

Nous résumons par le théorème:

Théorème 2: Toute géodésique $g$ qui ne passe pas par un point $P$ d'une variété de courbure partout négative possède un point à distance minimale de $P$. A partir de ce point les deux branches de la courbe s'éloignent de $P$ de façon monotone vers l'infini.

Nous allons déduire du développement du cône géodésique de sommet $P$ et de directrice $g$ d'autres conséquences.

\section{La somme des angles d'un quadrilatère géodésique dans une espace simplement connexe de courbure partout négative}

Théorème 3: La somme des angles d'un triangle géodésique dans un espace simplement connexe de courbure partout négative est inférieure à deux droits.

On entend par angle de deux rayons géodésiques évidemment la détermination de cet angle positive et inférieure à deux droits.

Le théorème a souvent été démontré, mais pour des triangles suffisamment petits ${ }^{26}$ ).

Démonstration: Soit $A B C$ un triangle géodésique quelconque. Nous considérons $A$ comme pôle et nous construisons le cône géodésique de sommet $A$ dont la géodésique $B C$ est une directrice. Nous développons ce cône comme nous l'avons fait dans 8 , II, l'équation de la géodésique $g=B C$ est:

d'où l'on tire:

$$
\frac{d \psi}{d \varphi}=-G_{r}<-1
$$

$$
\psi_{1}-\psi_{2}>\varphi_{2}-\varphi_{1}
$$

Or $\psi_{2}$ c'est l'angle $\gamma, \psi_{1}$ est le supplément de l'angle $\beta$ du triangle $A B C, \varphi_{2}-\varphi_{1}$ étant obtenu par un développement est plus grand que l'angle $\alpha$ du même triangle. On trouve:

c'est-à-dire:

$$
\pi-\beta-\gamma>\varphi_{2}-\varphi_{1}>\alpha,
$$

Ce qu'il fallait démontrer.

$$
\alpha+\beta+\gamma<\pi
$$

26) Voir par exemple ,Leçons“" p. 234. 
Nous sommes en mesure de démontrer facilement le théorème:

Théorème 4: La somme des angles d'un quadrilatère géodésique dans un espace simplement connexe est inférieure à quatre droits.

Démonstration: Nous traçons la ligne géodésique $B D$, et ainsi nous obtenons les deux triangles géodésiques $A B D$ et $B C D$. Pour ces deux triangles nous pouvons appliquer le théorème 3. Soient $\alpha^{\prime}, \beta^{\prime}, \delta^{\prime}$ les angles du triangle $A B D$ et $\beta^{\prime \prime}, \gamma, \delta^{\prime \prime}$ les angles du triangle $B C D$ :

d'où :

$$
\begin{array}{r}
\alpha+\beta^{\prime}+\quad \delta^{\prime}<\pi \\
\beta^{\prime \prime}+\gamma+\delta^{\prime \prime}<\pi
\end{array}
$$

$$
\alpha+\left(\beta^{\prime}+\beta^{\prime \prime}\right)+\gamma+\left(\delta^{\prime}+\delta^{\prime \prime}\right)<2 \pi .
$$

Or l'angle $A B C$ est inférieur à la somme des angles $A B D$ et $C B D$, ou éventuellement égal à cette somme:

de même:

$$
\beta^{\prime}+\beta^{\prime \prime} \leqslant \beta
$$

d'où l'on déduit:

$$
\delta^{\prime}+\delta^{\prime \prime} \leqslant \delta
$$

$$
\alpha+\beta+\gamma+\delta<2 \pi
$$

\section{Certaines isométries des espaces simplement connexes de courbure partout négative}

On peut considérer le groupe fondamental d'un espace de Riemann comme groupe d'isométries de l'espace simplement connexe de recouvrement comme nous l'avons vu dans 2, II. Les isométries de ce groupe sont des isométries sans points fixes. C'est pourquoi nous allons étudier les isométries possibles d'un espace simplement connexe de courbure partout négative sans aucun point fixe. En particulier, nous étudierons les isométries qui transforment une géodésique en elle-même en induisant une translation sur cette géodésique. Ces isométries sont analogues aux translations de l'espace hyperbolique. Nous les appellerons des ,translations".

Théorème 5*: Toute isométrie d'un espace simplement connexe de courbure partout négative ne transforme pas plus d'une géodésique en elle-même.

Démonstration: Supposons qu'une isométrie transforme les géodésiques $g_{1}$ et $g_{2}$ en elles-mêmes et soient $A_{1}$ et $A_{2}$ deux points situés respec- 
tivement sur $g_{1}$ et $g_{2}$, soit encore $g$ la géodésique $A_{1} A_{2}$. L'isométrie fait correspondre à $A_{1}, A_{2}$ et $g$, les points $A_{1}^{\prime}, A_{2}^{\prime}$ et la géodésique $g^{\prime}\left(A_{1}^{\prime}\right.$ et $A_{2}^{\prime}$ se trouvent respectivement sur $g_{1}$ et $\left.\mathrm{g}_{2}\right)$. Comme $g_{1}$ se transforme en elle-même par l'isométrie, celle-ci fera correspondre à l'angle $A_{2} A_{1} A_{1}^{\prime}$ l'angle congruent $A_{2}^{\prime} A_{1}^{\prime} A_{1}^{\prime \prime}$. De même l'angle $A_{1} A_{2} A_{2}^{\prime}$ est congruent à l'angle $A_{1}^{\prime} A_{2}^{\prime} A_{2}^{\prime \prime}$, de telle sorte que la somme des angles du quadrillatère géodésique $A_{1} A_{2} A_{2}^{\prime} A_{1}^{\prime}$ est égale à quatre droits, ce qui est impossible d'après le théorème 4 .

Théorème $6^{*}:$ Si une isométrie a est échangeable avec une autre isométrie $b$, et que a transforme une géodésique $g$ en elle-même, alors $b$ transforme aussi $g$ en elle-même.

Démonstration: Je prétends qu'avec les hypothèses de l'énoncé, la géodésique $b(g)$ est transformée en elle-même par l'isométrie $a_{2}$ en effet:

$$
a(b(g))=a b(g)=b(a(g))=b(g) .
$$

D'après le théorème $5^{*}$, cette géodésique est donc identique à $g$.

Nous allons maintenant donner quelques propriétés des groupes discontinus d'isométrie.

Théorème 7*: Les sous-groupes abéliens d'un groupe discontinu d'isométries sans points fixes qui contiennent au moins une "translation" sont des groupes cycliques.

En effet, d'après ce qui précède, nous savons que tous les éléments d'un groupe abélien qui contiennent une ,,translation“ sont des „,translations" sur la même géodésique. Comme le groupe est discontinu et que ses éléments n'admettent pas de points doubles, alors le groupe est déterminé complètement par le groupe de translations des points de la géodésique fixe commune et ce groupe est un groupe cyclique.

Si le groupe d'isométrie ne contient que des translations, alors tout sousgroupe abélien du groupe d'isométrie est un groupe cyclique.

Il est important d'autre part de connaître le domaine fondamental d'un groupe cyclique infini de ",translations".

La chose est, dans le cas des espaces de courbure partout négative, particulièrement simple. Nous savons en effet (théorème 2) que par un point extérieur on ne peut mener qu'une géodésique qui coupe à angle droit une géodésique donnée.

Soit $a$ la ,translation“ qui engendre le groupe cyclique, et $g$ la géodé- 
sique fixe qui correspond à $a, P$ est un point quelconque de $V_{n}$. Menons par $P$ la géodésique $n$ normale à $g$, et soit $Q$ le pied de la normale. La ,translation" $a$ fait correspondre au point $Q$ un point $a(Q)$ sur $g$, à la normale $n$ une géodésique $a(n)$ normale à $g$, à $P$ un point sur la géodésique $a(n)$ tel que la distance $a(P) a(Q)$ soit égale à la distance $P Q$. D'après ce qui précède, on voit facilement que le domaine fondamental est le domaine compris entre deux variétés géodésiques normales à $g$ (ces variétés géodésiques n'ont aucun point commun) et passant respectivement par les points $S$ et $a(S)$, où $S$ est un point quelconque de $g$. Ce domaine fondamental n'est pas borné et nous déduisons le théorème:

Théorème 8*: Le domaine fondamental d'un groupe cyclique de „translations" n'est pas borné.

\section{Les groupes fondamentaux des espaces de Riemann de courbure partout négative}

Dans 2, II nous avons vu que le groupe fondamental d'une variété non simplement connexe peut être considéré comme un groupe d'isométries de l'espace simplement connexe de recouvrement. Une géodésique fermée du type d'homotopie $a$ correspond dans l'espace simplement connexe de recouvrement à une ligne géodésique invariante par rapport à l'isométrie $a$. Les théorèmes $5^{*}, 7^{*}$ et $8^{*}$ qui concernent les groupes d'isométrie de l'espace simplement connexe de recouvrement deviennent alors quand on les considère comme des théorèmes sur les groupes fondamentaux :

Théorème 5: Dans un espace de courbure partout négative, il existe au plus une géodésique fermée de chaque type d'homotopie.

Théorème 7: Si un sous-groupe abélien du groupe fondamental d'un espace de courbure partout négative contient un élément pour lequel il correspond une géodésique fermée, ce sous-groupe est cyclique.

Et enfin:

Théorème 8: Le groupe fondamental d'une variété fermée de courbure partout négative n'est pas un groupe cyclique.

Dans le cas particulier des espaces fermés, le lemme 1 de 4, II assure l'existence d'une géodésique fermée correspondant à chaque classe d'homotopie et le théorème 7 devient:

Théorème 9: Tout sous-groupe abélien du groupe fondamental d'un espace fermé de courbure partout négative est un groupe cyclique. 
Des théorèmes 8 et 9 nous pouvons déduire le théorème 10:

Théorème 10: Le groupe fondamental d'une variété fermée de courbure partout négative n'est pas un groupe abélien.

Une conséquence du théorème 10 c'est que le tore de $n$ dimensions ne saurait être recouvert d'une métrique de courbure partout négative. $\mathrm{On}^{1}$ ) avait déjà démontré le fait pour le cas de la courbure constante négative, c'est-à-dire pour le cas des formes spatiales hyperboliques. Mais les théorèmes 8 et 9 permettent de montrer davantage, par exemple que le produit topologique, d'un cercle et d'une surface de genre 2 ne peut pas être recouvert d'une métrique de courbure par tout négative. En général aucun produit topologique de deux variétés topologiques fermées ne peut être métrisé par une métrique de courbure partout négative (théorème 9).

Remarque: D'après 7, II nous savons que tous les éléments $d u$ groupe fondamental d'un espace de courbure partout négative sont d'ordre infini. En effet, la variété simplement connexe de tout espace de courbure partout négative est homéomorphe à l'espace euclidien.

\section{Chapitre quatrième}

\section{Les espaces de courbure partout positive}

\section{La généralisation d'un théorème de Bonnet}

Théorème 11: Un espace complet dont la courbure est, pour tout élément plan, supérieure à un nombre positif $k$ est un espace fermé dont le diamètre est inférieur $\grave{a} \frac{\pi}{\sqrt{k}} \cdot{ }^{3}$ ), ${ }^{5}$ )

Démonstration: Il s'agit de montrer que si $A$ et $B$ sont deux points quelconques de l'espace, l'arc géodésique minimal qui les joint (cet arc existe puisque l'espace est complet) est de longueur inférieure à $\frac{\pi}{\sqrt{k}}$. Soit $g$ l'arc minimal $A B$ et $R$ un ruban obtenu en déplaçant par parallélisme un vecteur perpendiculaire à $g$. Nous pouvons construire dans le voisinage de $g$ une surface $V_{2}$ qui contienne le ruban $R$. D'après le lemme de Synge nous savons que la courbure gaussienne de la surface $V_{2}$ le long de $g$ est égale à la courbure riemannienne de $V_{n}$ pour le même élément plan tangent à $V_{2}$ le long de $g$, cette courbure est donc aussi supérieure à $k$. Nous savons alors que, déjà pour les variations de la géodésique sur $V_{2}$, un arc qui serait plus long que $\frac{\pi}{\sqrt{k}}$ ne serait pas un arc géodésique minimal. 
Remarque: La courbure d'un espace fermé de courbure partout positive possède un certain minimum positif. En effet, l'ensemble des éléments plans d'un espace fermé est un ensemble compact, et la courbure est une fonction continue des éléments plans. Le minimum de la courbure est donc atteint pour un certain élément plan. Ce minimum est done positif.

Théorème 12: Le groupe fondamental d'une variété fermée de courbure partout positive est fini. ${ }^{9}$ )

Démonstration: D'après la remarque, la courbure est partout supérieure à une constante $k$. La courbure de l'espace simplement connexe de recouvrement $\bar{V}_{n}$ est, pour chaque élément plan, égale à la courbure de l'élément plan correspondant dans $V_{n}$, elle est donc aussi supérieure à $k . \bar{V}_{n}$ est donc aussi, d'après le théorème 11 , un espace fermé, done borné et le groupe fondamental ne saurait être infini.

\section{D'autres propriétés des espaces fermés de courbure partout positive}

Théorème 13: Un espace orientale fermé $V_{n}$ de courbure partout positive et de dimension paire est simplement connexe.

Ce théorème a été démontré par Synge.

Nous démontrerons d'abord le lemme:

Lemme: Dans un espace de courbure positive et de dimension paire il n'existe pas de géodésique fermée plus courte que toutes les lignes fermées voisines et telle que l'orientation soit conservée quand on la parcourt une fois.

Démonstration du lemme: Supposons qu'il existe une telle géodésique $g$, je prétends que nous pourrons construire une surface $V_{2}$ de courbure positive dans le voisinage de $g$.

Choisissons un point quelconque $P$ de $g$ et en ce point un vecteur $\mathfrak{v}$ perpendiculaire à $g$ que nous transportons parallèlement à lui-même le long de $g$. Quand, après avoir décrit la courbe $g$, nous sommes revenus au point $P$, le vecteur $\mathfrak{v}$ a pris la position $\mathfrak{v}^{\prime}$. Le vecteur $\mathfrak{v}^{\prime}$ ne coïncide en général pas avec le vecteur $\mathfrak{v}$, mais en tout cas, $\mathfrak{v}^{\prime}$ est perpendiculaire à $g$. En $P$ nous avons construit une certaine correspondance $\left(\mathfrak{v}, \mathfrak{v}^{\prime}\right)$ entre les vecteurs perpendiculaires à $g$. Cette correspondance est orthogonale, ce qui est évident à cause des propriétés du déplacement parallèle. Or l'espace des vecteurs perpendiculaires à $g$ au point $P$ est un espace euclidien à $n-1$ dimensions et la correspondance $\left(\mathfrak{v}, \mathfrak{v}^{\prime}\right)$ est une 
transformation orthogonale de cet espace laissant invariant le point $P$. Les transformations orthogonales d'un espace euclidien laissant invariant un point fixe sont des rotations accompagnées ou non de symétrie. Ici, comme nous supposons que l'orientation est conservée, il ne peut s'agir que de rotations.

Or les rotations autour d'un point $P$ d'un espace euclidien à un nombre impair de dimensions laissent invariante au moins une direction passant par $P$. Cela signifie qu'il existe au moins un vecteur $\mathfrak{y}_{0}$ invariant dans la transformation $\left(\mathfrak{v}, \mathfrak{v}^{\prime}\right)$.

Si nous déplaçons les vecteurs $\mathfrak{p}_{0}$ et $-\mathfrak{v}_{0}$ parallèlement à eux-mêmes le long de $g$, nous obtenons un ruban continu le long de $g$.

Nous construisons une portion de surface quelconque $V_{2}$ contenant ce ruban. Le long de $g$ la courbure $K_{0}$ de $V_{2}$ est positive (lemme de Synge). Nous allons montrer que, déjà pour les variations effectuées dans le champ $V_{2}, g$ ne saurait être une courbe fermée minimale.

En effet, construisons sur $V_{2}$ un réseau $(u, v)$ de coordonnées de Fermi, c'est-à-dire que les coordonnées d'un point voisin de $g$ sont données:

$v$ par la longueur de la géodésique (sur $V_{2}$ ) perpendiculaire à $g$.

$u$ par la longueur de l'arc de $g$, d'une origine fixe au pied de la perpendiculaire.

$g$ est alors donné par l'équation $v=0$.

Considérons comme ,,courbes aux variations“ les courbes fermées

$$
v=\text { const. }
$$

La formule (2) de la deuxième variation (Chapitre I) nous donne:

$$
L^{\prime \prime}(0)=\int_{0}^{8}\left(\dot{\eta}^{2}-K \eta^{2}\right) d u+\eta^{n^{\prime}} \mid
$$

où $s$ représente la longueur de $g$.

Or, dans notre cas, les coordonnées $(u, v)$ coïncident avec les coordonnées de Fermi, par conséquent $\eta=1, \dot{\eta}=0$, d'autre part le point $(s, 0)$ coïncide avec le point $(0,0)$, le second terme du second membre est done nul. Il reste:

$$
L^{\prime \prime}(0)=\int_{0}^{s}-K d u
$$

Si $K$ est positif, la deuxième variation est négative, il ne saurait s'agir d'un minimum. Nous avons donc démontré le lemme. 
Démonstration du théorème 13: Un espace fermé orientable non simplement connexe possède une géodésique fermée minimale pour chaque type d'homotopie, si l'on décrit l'une de ces géodésiques l'orientation est conservée. Or ceci d'après le lemme ne se produit jamais pour des espaces de courbure partout positive.

Du théorème 13 nous pouvons déduire le

Théorème 13': Un espace non orientable fermé de courbure partout positive et de dimension paire possède un groupe fondamental d'ordre 2.

Théorème 13": Un espace de courbure partout positive et de dimension paire est orientable ${ }^{27}$ ).

On démontre le théorème $13^{\prime \prime}$ à l'aide du lemme:

Lemme: Dans un espace de courbure partout positive et de dimension impaire il n'existe pas de géodésique fermée plus courte que les lignes fermées voisines et telle que l'orientation change lorsqu'on la parcourt une fois.

La démonstration est tout-à-fait analogue à celle du lemme précédent.

\section{Un théorème sur les espaces ouverts de courbure partout positive}

On sait peu de choses sur les espaces ouverts de courbure partout positive. Nous allons démontrer un théorème de Cohn-Vossen ${ }^{28}$ ).

Théorème 14: Les espaces ouverts de courbure partout positive ne peuvent avoir qu'une extrémité.

Nous établirons d'abord deux lemmes:

Lemme 1: A chaque géodésique $g$ passant par un point $P$ d'un espace de courbure partout positive on peut faire correspondre un nombre $r$ tel que si les points $Q$ et $R$ sont situés sur $g$ de part et d'autre de $P$ et que :

$$
P Q>r \quad P R>r,
$$

alors l'arc de géodésique $Q P R$ ne représente pas le plus court chemin $Q R$.

27) Il ne semble pas que Synge ait dómontré le théorème 13 .

$\left.{ }^{28}\right)$ La démonstration de Cohn-Vossen ${ }^{12}$ ) diffère un peu de la nôtre. Cohn-Vossen se sert de deux lemmes: 1. Tout espace qui possède plus d'une extrémité possède une „droite géodésique" (ligne géodésique qui représente toujours le plus court chemin entre deux quelconques de ses points). 2. Dans un espace de courbure partout positive, il n'existe pas de droite géodésique. On construit la droite géodésique du lemme 1 par un procédé de convergence, le lemme 2 est une conséquence immédiate de notre lemme 1. 
Démonstration du lemme 1: Choisissons par $P$ une direction $\mathfrak{v}$ quelconque, perpendiculaire à la géodésique $g$ et transportons cette direction par parallélisme le long de $g$. Nous obtenons ainsi un ruban à deux dimensions le long de $g$. D'après le lemme de Synge, il correspond à chaque point de $g$ une certaine courbure $K_{0}$ du ruban et cette courbure est positive. Examinons la solution de l'équation différentielle:

$$
y+K_{0} y=0
$$

définie par les conditions initiales: $y(P)=1, \dot{y}(P)=0$.

Choisissons deux points $L$ et $M$ de part et d'autre de $P$ à la distance arbitraire $t$ de $P$, mais suffisamment près de $P$ pour que $y$ soit positif entre $L$ et $M$. Comme, dans l'intervalle $L M$, la concavité de la courbe $y=y(u)$ est tournée vers le bas, nous aurons: $\dot{y}(L)>0$ et $\dot{y}(M)<0$. Les tangentes à la courbe en $L$ et $M$ coupent l'axe des $u$ en $T^{\prime}$ et en $U^{\prime}$. Comme $K_{0}$ est positif, la courbe coupe l'axe des $u$ au moins en un point $T$ compris entre $T^{\prime}$ et $P$, et en un point $U$ compris entre $P$ et $U^{\prime}$. Nous pouvons maintenant construire entre $T^{\prime}$ et $U^{\prime}$ (par exemple) une portion de surface $V_{2}$ qui admette le ruban $R$ entre ces deux points. $g$ est encore une géodésique pour la surface et la courbure de $V_{2}$ le long de $g$ est $K_{0}$. L'équation (1) est l'équation de Jacobi pour la surface $V_{2}$ le long de $g$. Et nous venons de montrer qu'il existe une solution de cette équation dont deux racines $T$ et $U$ sont de part et d'autre de $P$. Tout arc qui contient l'arc $T U$ n'est donc pas un arc minimal déjà pour les variations sur $V_{2}$. On peut choisir par exemple $r$ comme la plus grande des deux longueurs (sur $g$ ) $P T^{\prime}$ et $P U^{\prime}$. Toute valeur plus grande jouit naturellement de la même propriété, qui est celle énoncée dans le lemme 1.

Remarque: Les distances $P T^{\prime}$ et $P U^{\prime}$ qui nous ont servi à apprécier $r$ ne dépendent que de la courbure dans le voisinage $L M$ de $P$ choisi arbitrairement. Si l'on remplaçait la courbure par une courbure plus faible et qu'on fît la même construction, alors les distances correspondantes $P T^{\prime \prime}$ et $P U^{\prime \prime}$ seraient plus grandes respectivement que $P T^{\prime}$ et $P U^{\prime}$ comme le montre le théorème de comparaison de Sturm. Soit par exemple $k_{0}$ un nombre positif qui soit une borne inférieure de la courbure dans l'intervalle $L M$. Nous obtenons alors comme solution de l'équation:

et nous trouvons:

$$
\begin{gathered}
\ddot{y}+k_{0} y=0 \\
y=\cos \left(\sqrt{k_{0}} u\right)
\end{gathered}
$$

$$
P T^{\prime \prime}=P U^{\prime \prime}=t+\frac{1}{\sqrt{k_{0}}} \cdot \operatorname{ctg}\left(\sqrt{k_{0}} t\right) .
$$


Cette remarque permet de démontrer le lemme 2:

Lemme 2: Soit $D$ un domaine fini de la variété $V_{n}$. Il existe un nombre $r$ fixe qui jouit pour tout point $Q$ de $D$ et pour toutes les géodésiques passant par $Q$ de la propriété énoncée dans le lemme 1 par rapport à $P$.

Démonstration du lemme 2: Définissons le domaine $D_{t}$, c'est-à-dire le domaine des points de $V_{n}$ dont la distance à $D$ est inférieure ou égale à $t$. Nous choisissons $t$ arbitraire positif. $D_{t}$ est un domaine compact. La courbure a alors dans le domaine $D_{t}$ un minimum positif que nous appellerons $k_{0}$.

Si nous choisissons une géodésique passant par un point quelconque $Q$ de $D$, il est certain que le segment de géodésique de centre $Q$ et de longueur $2 t$ est tout entier dans le domaine $D_{t}$. Par conséquent tout le long de ce segment la courbure est supérieure à $k_{0}$. D'après la remarque il suffit de choisir:

$$
r=t+\frac{1}{\sqrt{k_{0}}} \operatorname{ctg}\left(\sqrt{k_{0}} t\right) .
$$

On voit que $r$ ne dépend pas du point choisi $Q$.

Démonstration du théorème 14: La démonstration se fait par l'absurde. Supposons que la variété ait plusieurs extrémités, alors il existe deux suites divergentes de points:

telles que les lignes joignant:

$$
\begin{aligned}
& P_{1}, P_{2}, \ldots, P_{m}, \ldots \\
& Q_{1}, Q_{2}, \ldots, Q_{m}
\end{aligned}
$$

$$
P_{1} \text { à } Q_{1}, P_{2} \text { à } Q_{2}, \ldots, P_{m} \text { à } Q_{m}, \ldots
$$

aient toutes des points communs avec un certain domaine $D$ compact. Nous sommes alors sûrs qu'en particulier les arcs géodésiques minimaux:

$$
P_{1} Q_{1}, P_{2} Q_{2}, \ldots, P_{m} Q_{m}, \ldots
$$

ont des points communs avec le domaine $D$. A ce domaine nous pouvons attribuer un nombre $r$ d'après le lemme 2. Comme les suites $P_{m}$ et $Q_{m}$ sont divergentes, il existe une paire de points $P_{m}, Q_{m}$ telle que la distance de chacun de ces points à $D$ soit supérieure à $r$, mais cela est impossible puisqu'alors l'arc géodésique minimal ne représenterait pas la plus courte distance $P_{m} Q_{m}$.

Exemple: Le produit topologique d'une sphère et d'une droite (homéomorphe à l'espace euclidien dont on a enlevé un point) ne saurait être recouvert d'une métrique de courbure partout positive. Cet espace possède en effet deux extrémités. 


\section{Les espaces à pôles de courbure partout positive}

Tous les espaces simplement connexes de courbure partout négative sont des espaces à pôles; il existe des espaces de courbure partout positive qui ont un pôle, comme le montre l'exemple de l'espace donné par l'élément linéaire:

$$
d s^{2}=\sum_{i=1}^{n}\left(d x^{i}\right)^{2}+\left(\sum_{i=1}^{n} x^{i} d x^{i}\right)^{2}
$$

On montre d'autre part qu'on peut aussi construire des surfaces ouvertes simplement connexes et de courbure partout positive qui ne contiennent aucun pôle. Dans le cas particulier des espaces à pôles on peut démontrer quelques théorèmes.

Théorème 15: L'ensemble des pôles d'une variété à pôle de courbure partout positive est borné. ${ }^{13}$ )

Démonstration: Soit $P$ un point quelconque de la variété. Nous montrerons qu'on peut trouver un nombre fixe $r$ qui jouit de la propriété:

Par un point quelconque $Q$ qui est à une distance de $P$ supérieure à $r$ il passe un rayon géodésique qui n'est pas un rayon géodésique minimal.

Cette distance $r$ est celle que le lemme $2(3, \mathrm{IV})$ attribue à l'ensemble constitué par le seul point $P$. Soit, en effet, un point $Q$ à une distance de $P$ supérieure à $r$, nous pouvons prolonger la géodésique $Q P$ au delà de $P$ d'une distance supérieure à $r$ en $R$. Le lemme $2(3, I V)$ indique que l'arc géodésique $Q P R$ n'est pas un arc géodésique minimal et que par conséquent tous les rayons géodésiques issus de $Q$ ne sont pas des rayons géodésiques minimaux.

Théorème 16: S'il existe dans un espace $V_{n}$ de courbure partout positive un point $P$ qui ne possède de points conjugués dans aucune direction, alors l'espace est homéomorphe à l'espace euclidien et le point $P$ est un pôle.

Démonstration: D'après ce qui a été exposé en 5, II, nous savons qu'une variété qui contient un point $P$ sans point conjugué dans aucune direction possède une variété simplement connexe de recouvrement $\bar{V}_{n}$ homéomorphe au $R_{n}$ et que les points $\bar{P}_{i}$ qui correspondent dans $\bar{V}_{n}$ à $P$ sont des pôles de $\bar{V}_{n}$. Ces points $\bar{P}_{i}$ ne sauraient être en nombre infini car alors ils formeraient un ensemble non borné (les points $\bar{P}_{i}$ ne peuvent avoir de point d'accumulation) ce qui est en contradiction avec le théorème 15 appliqué à $\bar{V}_{n}$. Ils ne sauraient, d'autre part, être en nombre fini $(\neq 1)$ car alors le groupe fondamental serait lui aussi fini ce qui est en contradiction avec les remarques de 7 , II appliquées à $\bar{V}_{n}$ homéomorphe au $R_{n}$. 
Nous allons démontrer au sujet de l'allure des géodésiques des propriétés analogues à celles qui sont énoncées par le théorème 3. Nous utiliserons les lemmes 2 et 3 grâce auxquels nous avons démontré ce théorème et il nous faut démontrer un lemme analogue au lemme 1.

Lemme: Soit, dans un espace de courbure partout positive, un rayon géodésique $g$ issu d'un point $P$; s'il existe une surface $V_{2}$ contenant $g$, telle que la solution de l'équation de Jacobi:

$$
\ddot{y}+K_{0} y=0 \quad\left(K_{0} \text { courbure de } V_{2} \text { le long de } g\right)
$$

qui satisfait aux conditions initiales: $y(P)=0, \dot{y}(P)=1$ ait en un point une dérivée négative ou nulle, alors $g$ n'est pas un rayon géodésique minimal.

Démonstration du lemme: Si la dérivée $y$ est en un point négative ou nulle, il existe un premier point $Q$ pour lequel elle est nulle, et $y$ est positif entre $P$ et $Q$.

La surface $V_{2}$, que nous appelons la surface $\mathrm{I}\left(K_{0}=K_{0 I}\right)$ peut être recouverte d'un système de coordonnées analogue à celui que nous avons introduit dans le chapitre I: La géodésique $g$ est la courbe $v=0$. Les courbes $u_{\mathrm{I}}=u_{0}$ sont des courbes orthogonales à $g$ et $u_{\mathrm{I}}$ mesure l'arc sur la courbe $g$ à partir par exemple de l'origine $P$. La ligne $v_{\mathrm{I}}=v_{0}$ est obtenue en reportant sur les lignes $u_{\mathrm{I}}=u_{0}$ les distances $y_{\mathrm{r}}(u) v_{0}$. Nous avons ainsi défini un système de coordonnées $u, v$ (singulier en $P$ ) dans le voisinage de la géodésique $g$.

Au point $Q$ la ligne $u=u(Q)$ possède une tangente perpendiculaire à $g$ que nous déplaçons parallélement à elle-même le long de $g$. La courbure du ruban ainsi obtenu est positive (lemme de Synge), nous désignerons cette courbure par $K_{0 \mathrm{II}}$.

Choisissons un point $R$ au delà du segment $P Q$ suffisamment près de $Q$ pour que la solution de l'équation:

$$
\ddot{y}_{\mathrm{II}}+K_{\text {oII }} y_{\mathrm{II}}=0
$$

déterminée par les conditions initiales: $y_{\mathrm{II}}(R)=e>0, \dot{y}_{\mathrm{II}}(R)=0$ soit positive en $Q$. Nous donnerons alors au nombre $e$ une valeur telle que: $y_{1}(Q)=y_{\mathrm{II}}(Q)$.

Au point $R$ et à la géodésique $g$ correspond un nombre $r$ (lemme 1, 3, IV) tel que le premier point $S$ d'intersection de la courbe II avec l'axe des $u$ à droite de $R$, soit à une distance de $R$ inférieure à $r$. Nous pouvons alors construire une surface II contenant le ruban II entre $Q$ et $R$, ainsi que la courbe $u_{\mathrm{I}}=u_{\mathrm{I}}(Q)$. 
Sur la surface II nous construisons un système de coordonnées analogue à celui de la surface I. Les lignes $u_{\mathrm{II}}=u_{0}$ sont des lignes normales à $g$, et l'on choisit ces courbes de telle sorte que la ligne $u_{\mathrm{TI}}=u_{\mathrm{II}}(Q)$ coïncide avec la ligne $u_{\mathrm{I}}=u_{\mathrm{I}}(Q)$. On obtient les lignes $v_{1 \mathrm{I}}=v_{0}$ en reportant sur les lignes $u_{\Pi}=u_{0}$ la distance $y_{\mathrm{II}}\left(u_{0}\right) v_{0}$.

Enfin, nous construisons la famille de courbes définie de $P$ à $S$ par les conditions:

De $P$ à $Q$ une courbe de la famille est une courbe $v_{\mathrm{I}}=v_{0}$ sur la surface $\mathrm{I}$, qui se continue sur la surface II, par la courbe $v_{I I}=v_{0}$.

(Les deux arcs de courbe se raccordent vraiement à cause de la construction de la surface II.)

Nous pouvons calculer la deuxième variation de la longueur de la courbe $g$ pour cette famille de courbes, cette variation est la somme de la variation $L_{\mathrm{I}}^{\prime \prime}(0)$ de $P$ à $Q$ sur la surface $I$ et de la variation $L_{1 \mathrm{II}}^{\prime \prime}(0)$ de $Q$ à $S$ sur la surface II.

La formule (1) de $3, \mathrm{I}$ :

$$
\begin{aligned}
& L_{\mathrm{I}}^{\prime \prime}(0)=\int_{u_{P}}^{u_{Q}}\left(\dot{\eta}_{\mathrm{I}}^{2}-K_{0 \mathrm{I}} \eta_{\mathrm{I}}^{2}\right) d u+\left.\eta_{\mathrm{I}}^{n^{\prime}}\right|_{u_{P}} ^{u_{Q}} \\
& L_{\mathrm{II}}^{\prime \prime}(0)=\int_{u_{Q}}^{u_{S}}\left(\dot{\eta}_{\mathrm{II}}^{2}-K_{0 \mathrm{II}} \eta_{\mathrm{II}}^{2}\right) d u+\left.\eta_{\mathrm{II}}^{n^{\prime}}\right|_{u_{Q}} ^{u_{S}} .
\end{aligned}
$$

Une intégration partielle nous donne:

$$
\begin{aligned}
& L_{\mathrm{I}}^{\prime \prime}(0)=-\int_{u_{P}}^{u_{Q}} \eta_{\mathrm{I}}\left(\ddot{\eta}_{\mathrm{I}}+K_{0 \mathrm{I}} \eta_{\mathrm{I}}\right) d u+\left.\eta \dot{\eta}\right|_{u_{P}} ^{u_{Q}}+\left.\eta_{\mathrm{I}}^{n^{\prime}}\right|_{u_{P}} ^{u_{Q}} \\
& L_{\mathrm{II}}^{\prime \prime}(0)=-\int_{u_{Q}}^{u_{S}} \eta_{\mathrm{II}}\left(\ddot{\eta}_{\mathrm{II}}+K_{0 \mathrm{II}} \eta_{\mathrm{II}}\right) d u+\left.\eta \dot{\eta}\right|_{u_{Q}} ^{u_{S}}+\left.\eta_{\mathrm{II}}^{n^{\prime}}\right|_{u_{Q}} ^{u_{S}} .
\end{aligned}
$$

Or ici la valeur de $\eta$ est $y$. On voit que les intégrales sont nulles puisque $\eta_{\mathrm{I}}$ et $\eta_{\mathrm{II}}$ satisfont à des équations de Jacobi. Comme toutes les courbes de la famille passent par les points $P$ et $S$ :

Il reste donc:

$$
\begin{aligned}
& \eta_{\mathrm{I}}, \eta_{\mathrm{I}}^{n^{\prime}} \text { sont nuls au point } P \text {. } \\
& \eta_{\mathrm{II}}, \eta_{\mathrm{II}}^{n^{\prime}} \text { sont nuls au point } S .
\end{aligned}
$$

$$
L^{\prime \prime}(0)=L_{\mathrm{I}}^{\prime \prime}(0)+L_{\mathrm{II}}^{\prime \prime}(0)=\left(\eta_{1} \dot{\eta}_{\mathrm{I}}-\eta_{\mathrm{II}} \dot{\eta}_{\mathrm{II}}\right)+\left(\eta_{\mathrm{I}}^{n^{\prime}}-\eta_{\mathrm{II}}^{n^{\prime}}\right) .
$$

Les valeurs $\eta, \dot{\eta}, \eta^{n^{\prime}}$ étant prises au point $Q$. 
Or les valeurs $\eta_{\mathrm{I}}^{n^{\prime}}$ et $\eta_{\mathrm{II}}^{n^{\prime}}$ ne dépendent que de la courbe $u=u(Q)$ qui est la même pour les deux surfaces I et II. D'autre part:

d'où :

$$
y_{\mathrm{I}}(Q)=y_{\mathrm{II}}(Q), \quad \dot{y}_{\mathrm{I}}(Q)=0 \quad y_{\mathrm{II}}(Q)>0
$$

$$
L^{\prime \prime}(0)<0
$$

ce qui est incompatible avec le fait que $g$ soit un rayon géodésique minimal relatif. Le lemme est donc démontré.

Nous sommes en mesure de démontrer le théorème:

Théorème 17: Toute géodésique $g$ qui ne passe pas par le pôle $P$ d'une variété à pôle de courbure partout positive possède un point à distance minimale de $P$. A partir de ce point les deux branches de la courbe s'éloignent de $P$ de façon monotone vers l'infini.

Démonstration: Soit $g$ une géodésique quelconque ne passant pas par $P$, nous pouvons faire, comme dans 8 , II, la construction du cône géodésique de sommet $D$ et de directrice $g$ sur lequel nous introduisons un système de coordonnées géodésiques, l'élément d'arc est donné alors par la forme:

$$
d s^{2}=d r^{2}+G^{2} d \varphi^{2}
$$

et le lemme que nous venons de démontrer montre que quelle que soit la géodésique $g$ la quantité $G_{r}$ est positive (tout rayon géodésique issu de $P$ est un rayon géodésique minimal), ce qui est équivalent au lemme 1 $(2, \mathrm{III})$.

Nous pouvons alors appliquer les lemmes 2 et 3 de 2 , III et nous trouvons le théorème 17 .

En particulier nous avons démontré que dans un espace à pôle de courbure partout positive il n'existe pas de géodésique fermée.

(Reçu le 5 juillet 1942.) 


\section{CURRICULUM VITAE}

Né le 28 mai 1916 à Neuchâtel, j'ai suivi les écoles primaires et secondaires à La Chaux-de-Fonds. Baccalauréat classique en juillet 1934. Dès octobre 1934 étudiant à l'École Polytechnique Fédérale dans la section pour l'enseignement des mathématiques et de la physique, j'obtins le diplôme en octobre 1938. De décembre 1938 à septembre 1939, pratiquant chez M. le Professeur GONSETH. D'octobre 1939 à septembre 1940, assistant chez M. le Professeur KOLLROS. Dès octobre 1940, assistant chez M. le Professeur Gonseth.

Le présent travail a été fait sous la direction de M. le Professeur HOPF, auquel je dois toute ma reconnaissance.

Zurich, le 22 mars 1942.

A. Preissmann. 